\title{
Task Space Trajectory Tracking Control of Robot Manipulators with Uncertain Kinematics and Dynamics
}

\author{
Xichang Liang, Yi Wan, and Chengrui Zhang \\ Key Laboratory of High Efficiency and Clean Mechanical Manufacture, Ministry of Education, School of Mechanical Engineering, \\ Shandong University, 17923 Jingshi Road, Jinan City, Shandong, China
}

Correspondence should be addressed to Yi Wan; wanyi@sdu.edu.cn

Received 19 July 2017; Revised 13 October 2017; Accepted 18 October 2017; Published 11 December 2017

Academic Editor: Jean Jacques Loiseau

Copyright (C) 2017 Xichang Liang et al. This is an open access article distributed under the Creative Commons Attribution License, which permits unrestricted use, distribution, and reproduction in any medium, provided the original work is properly cited.

To improve the tracking precision of robot manipulators' end-effector with uncertain kinematics and dynamics in the task space, a new control method is proposed. The controller is based on time delay estimation and combines with the nonsingular terminal sliding mode (NTSM) and adaptive fuzzy logic control scheme. Kinematic parameters are not exactly required with the consideration of kinematic uncertainties in the controller. No dynamic models or numerous parameters of the robot manipulator system are required with the use of TDE. Thus, the controller is simple structure and suitable for practical applications. Furthermore, errors caused by time delay estimation are compensated by the adaptive fuzzy nonsingular terminal sliding mode scheme. The simulation is performed on a 2-DOF robot manipulator with three cases in the task space. The results show that the proposed controller provides faster convergence rate and higher tracking precision than TDE based NTSM and improved TDE based NTSM controller.

\section{Introduction}

Robot manipulators play an important role in the industry automation field in recent years [1-3]. One of the irreplaceable capabilities of robot manipulations is the high accuracy and high-speed performance of trajectory tracking. Therefore, engineers have sought to realize their automatic control by various control methods, such as sliding mode control (SMC) [4-7], adaptive control [8], neural network methods [9], and fuzzy logic control [10]. The automatic control of robot manipulators presents a unique challenge from the control aspect, which is caused by inherent large nonlinearities and external uncertainties in system dynamics. Generally, to obtain a satisfactory control performance, the above control methods are mostly either model-free types introducing numerous parameters or model-based types requiring nominal model [11]. However, robot manipulators, similar to many other mechanical systems, are complex and nonlinear. It is difficult to establish an accurate dynamic model of a robot manipulator system, which limits the practical applications of the control methods.
It is noteworthy that time delay estimation (TDE) provides a simple way to solve the above problems. The main idea of the TDE is to estimate unknown dynamics and disturbances by intentionally using time delayed information [12]. The main advantage of the TDE is the mitigation of tedious modeling burden of complex system [13]. Due to its advantages, TDE has been widely used in the design process of various controllers and provides satisfactory results [1416]. Lee et al. proposed an adaptive robust controller using TDE and adaptive integral sliding mode control, which was proved to be robust, chattering-free, and highly accurate [17]. Roy et al. proposed a new adaptive robust control strategy with time delay control to remove prerequisite of system model and to alleviate the over- or underestimation problems of the switching gain [13]. A systematic method was proposed using time delay estimation to simplify the tune process of fuzzy PID controller by Kim et al. [18].

Controllers designed based on TDE are typically composed of two elements. One element is the TDE element, which cancels nonlinear dynamics. The other element is an injecting element, which endows desired error dynamics. 
Linear error dynamics are widely used as the desired error dynamics in TDE based controllers, such as time delay control (TDC) [19]. Through extensive research, controllers based on TDC have been developed to improve the control performance. To suppress estimation error in TDC, fuzzy logic system was introduced in TDC as the third element by Bae et al., and satisfactory results were obtained [20]. To realize the automatic tuning of TDC parameters, adaptive time delay control was proposed by Jin et al. [21] and Cho et al. [22], respectively. To improve the convergence rate and tracking precision, the nonlinear error dynamics such as nonsingular terminal sliding mode (NTSM) were used together with TDE by recent work $[23,24]$. Jin et al. employed this control method in the trajectory tracking of robot manipulators by simulations and experiments. The tracking results showed that the controller is highly accurate, model-free, simple to implement, and robust. However, there are two aspects to be improved. (1) Kinematic uncertainties are not considered in the controller design process. When the desired trajectory is planned in the task space such as Cartesian space, the Jacobian matrix from joint space to Cartesian space and the kinematic parameters of the robot manipulator are assumed exactly to be known. Satisfactory performance can be obtained in the task space without the feedback of the end-effector position with the assumption. But in most practical applications, the kinematics parameters may not be exactly known, which is caused by the interaction between manipulator and different environments and the imprecise measurements of physical parameters. (2) TDE can not eliminate the nonlinearities such as Coulomb friction perfectly. Those nonlinearities may cause TDE error, which reduces the precision of trajectory tracking [25-27].

In order to solve the above problems, a novel control method is proposed for trajectory tracking of robot manipulator's end-effector in the task space. The controller is based on TDE and combines with the NTSM and adaptive fuzzy logic control scheme. The contributions of this controller are listed as follows. (1) The kinematic uncertainties of the manipulator are taken into consideration without the prior knowledge of system. (2) No dynamic models of the robot manipulator system are required. (3) The adaptive fuzzy logic scheme is implemented as the third element to compensate the TDE error and eliminate the undesired chattering in NTSM. (4) The controller is easy to implement with simple structure, and it is suitable for practical applications.

\section{TDE Based NTSM Control with Kinematic and Dynamic Uncertainties}

In this section, the conventional TDE based NTSM controller is improved with the consideration of kinematic uncertainties. The control objective of this controller is to make the end-effector of manipulator follow a desired trajectory in the task space with unknown dynamics and uncertain kinematics.

The dynamical equation of $n$-link robot manipulator can be shown as

$$
\mathbf{M}(\boldsymbol{\theta}) \ddot{\theta}+\mathbf{C}(\boldsymbol{\theta}, \dot{\theta}) \dot{\theta}+\mathbf{G}(\boldsymbol{\theta})+\mathrm{F}(\boldsymbol{\theta}, \dot{\theta})+\tau_{\mathrm{d}}=\tau,
$$

where $\boldsymbol{\theta}, \dot{\boldsymbol{\theta}}, \ddot{\boldsymbol{\theta}} \in \mathbf{R}^{n}$ are vector of position, velocity, and acceleration of the joints respectively; $\boldsymbol{\tau} \in \mathbf{R}^{n}$ denotes the actuator torque; and $\mathbf{M}(\boldsymbol{\theta}) \in \mathbf{R}^{n \times n}$ represents the inertia matrix; $\mathbf{V}(\boldsymbol{\theta}, \dot{\boldsymbol{\theta}}) \in \mathbf{R}^{n}$ stands for the Coriolis and centrifugal matrix; $\mathbf{G}(\boldsymbol{\theta}) \in \mathbf{R}^{n}$ is the gravitational vector; $\mathbf{F} \in \mathbf{R}^{n}$ is the friction term; and $\mathbf{D} \in \mathbf{R}^{n}$ denotes the disturbance torques.

Equation (1) can be rewritten as follows after defining a constant diagonal matrix $\overline{\mathbf{M}}$ :

$$
\overline{\mathbf{M}} \ddot{\boldsymbol{\theta}}+\mathbf{H}=\boldsymbol{\tau},
$$

where $\mathbf{H}=[\mathbf{M}(\boldsymbol{\theta})-\overline{\mathbf{M}}] \ddot{\boldsymbol{\theta}}+\mathbf{C}(\boldsymbol{\theta}, \dot{\boldsymbol{\theta}}) \dot{\boldsymbol{\theta}}+\mathbf{G}(\boldsymbol{\theta})+\mathbf{F}(\boldsymbol{\theta}, \dot{\boldsymbol{\theta}})+\boldsymbol{\tau}_{\mathrm{d}}$.

The trajectory of manipulator end-effector in the task space can be expressed as

$$
\mathbf{x}=\mathbf{h}(\boldsymbol{\theta}),
$$

where $\mathbf{h}(\boldsymbol{\theta})$ is the transformation relationship between the task space and the joint space; $\mathbf{x}$ is the trajectory of endeffector in the task space, which can be measured by laser sensor or vision sensors. $\dot{\mathbf{x}}$ is the velocity in task space and it is related to $\dot{\boldsymbol{\theta}}$ in the joint space, which can be expressed as

$$
\dot{\mathbf{x}}=\mathbf{J}(\boldsymbol{\theta}) \dot{\boldsymbol{\theta}},
$$

where $\mathbf{J}(\boldsymbol{\theta})$ is the Jacobian matrix.

The differential of (4) can be expressed as

$$
\ddot{\mathbf{x}}=\mathbf{J}(\boldsymbol{\theta}) \ddot{\boldsymbol{\theta}}+\dot{\mathbf{J}}(\boldsymbol{\theta}) \dot{\boldsymbol{\theta}}
$$

It should be noted that the inverse of Jacobin matrix will be an ill conditioned matrix when the manipulator is at the vicinity of a singular configuration, and the singularity problem exists in the control of robot manipulators. Some methods have been proposed to solve this problem [28]. In this paper, it is assumed that the Jacobian matrix is nonsingular, and then (5) can be rewritten as

$$
\ddot{\boldsymbol{\theta}}=\mathbf{J}^{-1}(\boldsymbol{\theta})[\ddot{\mathbf{x}}-\dot{\mathbf{J}}(\boldsymbol{\theta}) \dot{\boldsymbol{\theta}}] .
$$

Substituting (6) into (2), it can be obtained as

$$
\boldsymbol{\tau}=\overline{\mathbf{M}} \mathbf{J}^{-1}(\boldsymbol{\theta})[\ddot{\mathbf{x}}-\dot{\mathbf{J}}(\boldsymbol{\theta}) \dot{\boldsymbol{\theta}}]+\mathbf{H} .
$$

Because the kinematic and dynamic parameters of robot manipulators are not exactly known in the practical applications, the parameters in (8) are substituted by the estimated value.

$$
\tau=\overline{\mathbf{M J}}^{-1}(\boldsymbol{\theta})[\ddot{\mathbf{x}}-\dot{\hat{\mathbf{J}}}(\boldsymbol{\theta}) \dot{\boldsymbol{\theta}}]+\widehat{\mathbf{H}}
$$

where $\widehat{\mathbf{H}}$ and $\widehat{\mathbf{J}}^{-1}(\boldsymbol{\theta})$ are estimated parameters of $\boldsymbol{H}$ and $\mathbf{J}^{-1}(\boldsymbol{\theta})$.

Replacing $\ddot{\mathbf{x}}$ by the acceleration of the desired trajectory $\ddot{\mathbf{x}}_{\mathbf{d}}$, the equivalent control input can be expressed as

$$
\tau_{\text {eq }}=\overline{\mathbf{M J}}^{-1}(\boldsymbol{\theta})\left[\ddot{\mathbf{x}}_{\mathbf{d}}-\dot{\hat{\mathbf{J}}}(\boldsymbol{\theta}) \dot{\boldsymbol{\theta}}\right]+\widehat{\mathbf{H}}
$$

To compensate the kinematic and dynamic uncertainties and guarantee the stability, the NTSM scheme $\boldsymbol{\tau}_{\mathrm{ntsm}}$ is implemented as

$$
\tau=\tau_{\text {eq }}+\tau_{\text {ntsm }}
$$


The NTSM scheme is designed by two steps. The first step is the design of a sliding surface, and the second step is the design of a control law to guarantee the existence of sliding mode.

Then, sliding mode surface is chosen as

$$
\mathbf{s}=\mathbf{e}+\mathbf{K} \dot{\mathbf{e}}^{\mathbf{p} / \mathbf{q}},
$$

where $\mathbf{e}$ is the tracking error between the desired position and actual position of the manipulator end-effector in the task space, and $\mathbf{e}=\mathbf{x}_{\mathbf{d}}-\mathbf{x} ; \mathbf{s}$ represents an $n \times 1$ vector; $K$ denotes an $n \times n$ defined positive constant matrix; $p$ and $q$ are positive odd integers and $1<p / q<2$.

To guarantee the existence of sliding mode, the NTSM scheme is designed as

$$
\tau_{\text {ntsm }}=\widehat{\mathbf{M J}}^{-1}(\boldsymbol{\theta})\left[\frac{\mathbf{q}}{\mathbf{p}} \mathbf{K}^{-1} \dot{\mathbf{e}}^{2-\mathbf{p} / \mathbf{q}}+\mathbf{K}_{\mathrm{sw}} \operatorname{sgn}(\mathbf{s})\right],
$$

where $\mathbf{K}_{\text {sw }}$ is a constant matrix to be designed.

Then, the control law can be expressed as

$$
\begin{aligned}
\tau= & \tau_{\text {eq }}+\tau_{\text {ntsm }}=\overline{\mathbf{M}}^{-1}(\boldsymbol{\theta}) \\
& \cdot\left[\ddot{\mathbf{X}}_{\mathbf{d}}-\dot{\hat{\mathbf{J}}}(\boldsymbol{\theta}) \dot{\boldsymbol{\theta}}+\frac{\mathbf{q}}{\mathbf{p}} \mathbf{K}^{-1} \dot{\mathbf{e}}^{2-\mathbf{p} / \mathbf{q}}+\mathbf{K}_{\mathrm{sw}} \operatorname{sgn}(\mathbf{s})\right]+\widehat{\mathbf{H}} .
\end{aligned}
$$

In this control law, $\widehat{\mathbf{H}}$ is the estimation of all the nonlinearities kinematic and dynamic uncertainties, which makes it difficult to establish model and identify parameters. TDE is used to estimate $\widehat{\mathbf{H}}$ in this controller, which can be expressed as

$$
\widehat{\mathbf{H}}=\mathbf{H}_{t-L}=\boldsymbol{\tau}_{t-L}-\overline{\mathbf{M}} \ddot{\theta}_{t-L},
$$

where $\bullet_{t-L}$ denotes the time delayed value of $\bullet$, and if the time delay $L$ is set as infinitesimally small, an estimation of $\mathbf{H}$ would be possible by TDE.

Then the output of the controller can be expressed as

$$
\begin{aligned}
\boldsymbol{\tau}= & \boldsymbol{\tau}_{\mathrm{eq}}+\boldsymbol{\tau}_{\mathrm{ntsm}}+\boldsymbol{\tau}_{\mathrm{tde}}=\overline{\mathbf{M}}^{-1}(\boldsymbol{\theta}) \\
& \cdot\left[\ddot{\mathbf{x}}_{\mathbf{d}}-\dot{\hat{\mathbf{J}}}(\boldsymbol{\theta}) \dot{\boldsymbol{\theta}}+\frac{\mathbf{q}}{\mathbf{p}} \mathbf{K}^{-1} \dot{\mathbf{e}}^{2-\mathbf{p} / \mathbf{q}}+\mathbf{K}_{\mathrm{sw}} \operatorname{sgn}(\mathbf{s})\right]+\boldsymbol{\tau}_{t-L} \\
& -\overline{\mathbf{M}}_{t-L} .
\end{aligned}
$$

Substituting controller equation (15) into dynamics equation (2), it can be obtained as

$$
\begin{aligned}
\overline{\mathbf{M}} \widehat{\mathbf{J}}^{-1}(\boldsymbol{\theta})\left[\ddot{\mathbf{X}}_{\mathbf{d}}-\dot{\hat{\mathbf{J}}}(\boldsymbol{\theta}) \dot{\boldsymbol{\theta}}+\frac{\mathbf{q}}{\mathbf{p}} \mathbf{K}^{-1} \dot{\mathbf{e}}^{2-\mathbf{p} / \mathbf{q}}+\mathbf{K}_{\mathbf{s w}} \operatorname{sgn}(\mathbf{s})\right] \\
+\overline{\mathbf{M}} \ddot{\boldsymbol{\theta}}+\mathbf{H}_{t-L}-\mathbf{H}=0 .
\end{aligned}
$$

In (6), the Jacobin matrix is substituted by the estimated value. It can be expressed as

$$
\ddot{\boldsymbol{\theta}}=\widehat{\mathbf{J}}^{-1}(\boldsymbol{\theta})[\ddot{\mathbf{x}}-\dot{\hat{\mathbf{J}}}(\boldsymbol{\theta}) \dot{\boldsymbol{\theta}}] .
$$

Substituting (17) into (16), it can be obtained as

$$
\ddot{\mathbf{e}}+\frac{q}{p} \mathbf{K}^{-1} \dot{\mathbf{e}}^{2-\mathbf{p} / \mathbf{q}}+\mathbf{K}_{\mathbf{s w}} \operatorname{sgn}(\mathbf{s})=\boldsymbol{\varepsilon},
$$

where $\boldsymbol{\varepsilon}=\widehat{\mathbf{J}}(\boldsymbol{\theta}) \overline{\mathbf{M}}^{-1}\left(\mathbf{H}_{t-L}-\mathbf{H}\right)$ and it is defined as TDE error. The TDE error is mainly caused by the finite time delay $L$ under nonlinearities such as Coulomb friction.

Lyapunov function is chosen to prove the stability of the system, which is expressed as

$$
V=0.5 \mathbf{s}^{T} \mathbf{s}
$$

Then, the derivative of $V$ is expressed as

$$
\begin{aligned}
\dot{V} & =\mathbf{s}^{T} \dot{\mathbf{s}}=\mathbf{s}^{T}\left(\dot{\mathbf{e}}+\frac{\mathbf{p}}{\mathbf{q}} \mathbf{K}^{\mathbf{p} / \mathbf{q}-1} \ddot{\mathbf{e}}\right)=\mathbf{s}^{T}\{\dot{\mathbf{e}} \\
& \left.+\frac{\mathbf{p}}{\mathbf{q}} \mathbf{K}^{\mathbf{p} / \mathbf{q}-1}\left[-\frac{\mathbf{q}}{\mathbf{p}} \mathbf{K}^{-1} \dot{\mathbf{e}}^{2-\mathbf{p} / \mathbf{q}}-\mathbf{K}_{\mathbf{s w}} \operatorname{sgn}(\mathbf{s})+\boldsymbol{\varepsilon}\right]\right\} \\
& =\mathbf{s}^{T} \frac{\mathbf{p}}{\mathbf{q}} \mathbf{K e}^{\mathbf{p} / \mathbf{q}-1}\left[\boldsymbol{\varepsilon}-\mathbf{K}_{\mathbf{s w}} \operatorname{sgn}(\mathbf{s})\right] .
\end{aligned}
$$

According to the study of Jin et al. [24], $\varepsilon$ is proved to be bounded. In (20), $\mathbf{p}$ and $\mathbf{q}$ are positive odd integers and $1<$ $\mathbf{p} / \mathbf{q}<2$; then $\dot{e}^{p_{i} / q_{i}-1}>0$ for $\dot{e} \neq 0$. To guarantee the stability of the system, $\mathbf{K}_{\mathbf{s w}}$ should be chosen as

$$
\mathbf{K}_{\mathrm{sw}}>|\varepsilon| .
$$

Then, $\mathbf{s}^{T}(\mathbf{p} / \mathbf{q}) \mathbf{K} \dot{\boldsymbol{e}}^{\mathbf{p} / \mathbf{q}-1}\left[\boldsymbol{\varepsilon}-\mathbf{K}_{\mathbf{s w}} \operatorname{sgn}(\mathbf{s})\right]<0$ and $\dot{V}<0$.

Thus, the controller considering the kinematic and dynamic uncertainties is proposed as (15). However, it still has two drawbacks. One is the chattering problem caused by sign function. Although the replacement of the sign function by saturation function can eliminate the chattering, it reduces the tracking precision $[29,30]$. The other problem is the TDE errors, which is shown in (18). TDE errors may cause the tracking error of the system.

\section{TDE Based AFNTSM Control with Kinematic and Dynamic Uncertainties}

In order to solve the above problems, adaptive fuzzy logic control scheme is used to eliminate the chattering and improve the tracking precision. In this section, the controller is presented and the stability is proved based on the Lyapunov method. The new controller structure is designed as

$$
\begin{aligned}
\boldsymbol{\tau}= & \boldsymbol{\tau}_{\mathrm{eq}}+\boldsymbol{\tau}_{\mathrm{fntsm}}+\boldsymbol{\tau}_{\mathrm{tde}} \\
= & \overline{\mathbf{M}}^{-1}(\boldsymbol{\theta})\left[\ddot{\mathbf{x}}_{\mathbf{d}}-\dot{\hat{\mathbf{J}}}(\boldsymbol{\theta}) \dot{\boldsymbol{\theta}}+\frac{\mathbf{q}}{\mathbf{p}} \mathbf{K}^{-1} \dot{\mathbf{e}}^{2-\mathbf{p} / \mathbf{q}}+\mathbf{k}+\boldsymbol{\rho} \mathbf{s}\right] \\
& +\boldsymbol{\tau}_{t-L}-\overline{\mathbf{M}} \ddot{\theta}_{t-L} .
\end{aligned}
$$

Figure 1 demonstrates the block diagram of the proposed controller. It is composed of three terms, including the equivalent control term, the adaptive fuzzy nonsingular terminal sliding mode term, and time delay estimation term. 


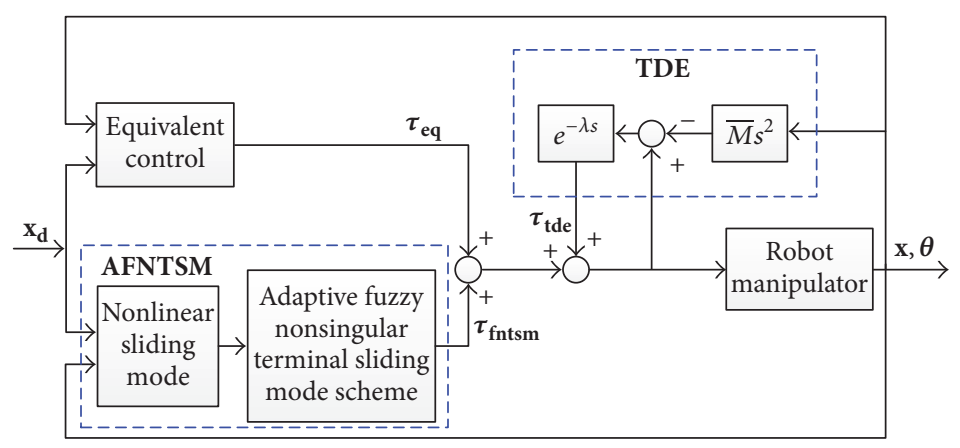

FIGURE 1: Block diagram of the proposed controller.

The main difference of controller equation (22) and equation (15) is the replacement of sign function by an adaptive fuzzy logic control scheme $\mathbf{k}+\boldsymbol{\rho}$ s, where $\mathbf{k}$ is the adaptive fuzzy logic controller and $\boldsymbol{\rho}$ s is its compensator. $\boldsymbol{\rho}$ is designed as a diagonal positive definite matrix and $\boldsymbol{\rho}=\operatorname{diag}\left[a_{1}+\sigma_{1}, \ldots, a_{i}+\right.$ $\left.\sigma_{i}, \ldots, a_{n}+\sigma_{n}\right]$, where $a_{i}$ is a positive constant and $\sigma_{i}$ is a positive value.

3.1. Fuzzy Logic Control Scheme. It is designed that $\mathbf{k}$ has the same sign as that of $s_{i}$. Substituting (22) into (20), it is obtained as

$$
\dot{V}=\mathbf{s}^{T} \frac{\mathbf{p}}{\mathbf{q}} \mathbf{K} \dot{\mathbf{e}}^{\mathbf{p} / \mathbf{q}-1}[\boldsymbol{\varepsilon}-\mathbf{k}-\boldsymbol{\rho} \mathbf{s}] .
$$

Because $(\mathbf{p} / \mathbf{q}) \mathbf{K} \dot{\mathbf{e}}^{\mathbf{p} / \mathbf{q}-1}>0$, the term $\mathbf{s}^{T}[\boldsymbol{\varepsilon}-\mathbf{k}-\boldsymbol{\rho} \mathbf{s}]$ in (23) is considered. When $\left|s_{i}\right|$ is large, it is expected that $\left|k_{i}\right|$ is large so that $\dot{V}$ can be a larger negative value. When $\left|s_{i}\right|$ is small, $\mathbf{s}^{T}[\boldsymbol{\varepsilon}-\mathbf{k}-\boldsymbol{\rho s}]$ is very small, which has little effect on the value of $\dot{V}$. Then, small $\left|k_{i}\right|$ is allowed to avoid chattering. When $\left|s_{i}\right|$ is zero, $\mathbf{s}^{T}[\boldsymbol{\varepsilon}-\mathbf{k}-\boldsymbol{\rho} \mathbf{s}]$ is zero and $\left|k_{i}\right|$ can be zero. From these analyses, the rule base is defined as

$$
\begin{aligned}
& \text { IF } s_{i} \text { is NB, THEN } k_{i} \text { is NB } \\
& \text { IF } s_{i} \text { is NS, THEN } k_{i} \text { is NS } \\
& \text { IF } s_{i} \text { is ZE, THEN } k_{i} \text { is ZE } \\
& \text { IF } s_{i} \text { is PS, THEN } k_{i} \text { is PS } \\
& \text { IF } s_{i} \text { is } \mathrm{PB}, \operatorname{THEN} k_{i} \text { is } \mathrm{PB} \text {, }
\end{aligned}
$$

where $s_{i}$ the input of fuzzy system and $k_{i}$ is the output of the system. They are partitioned into five fuzzy subsets: positive big $(\mathrm{PB})$, positive small (PS), zero (ZE), negative small (NS), and negative big (NB). They are Gaussian membership function defined as

$$
\mu_{A}\left(x_{i}\right)=\exp \left[-\left(\frac{x_{i}-\alpha}{\sigma}\right)^{2}\right],
$$

where the subscript $A$ denotes the fuzzy sets such as $\mathrm{PB}, \ldots, \mathrm{NB} ; x_{i}$ donates $s_{i}$ and $k_{i} ; \alpha$ is the center of $A$; and $\sigma$ is the width of $A$.
Choosing the product inference engine, singleton fuzzification, and center average defuzzification, then, $k_{i}$ can be written as

$$
k_{i}=\frac{\sum_{m=1}^{M} \gamma_{k_{i}}^{m} \mu_{A}^{m}\left(s_{i}\right)}{\sum_{m=1}^{M} \mu_{A}^{m}\left(s_{i}\right)}=\gamma_{k_{i}}^{T} \psi_{k_{i}}\left(s_{i}\right),
$$

where $\gamma_{k_{i}}=\left[\gamma_{k_{i}}^{1}, \ldots, \gamma_{k_{i}}^{m}, \ldots, \gamma_{k_{i}}^{M}\right]^{T}, \psi_{k_{i}}\left(s_{i}\right)=\left[\psi_{k_{i}}^{1}\left(s_{i}\right), \ldots\right.$, $\left.\psi_{k_{i}}^{m}\left(s_{i}\right), \ldots, \psi_{k_{i}}^{M}\left(s_{i}\right)\right]^{T}$, and $\psi_{k_{i}}^{m}\left(s_{i}\right)=\mu_{A^{m}}\left(s_{i}\right) / \sum_{m=1}^{M} \mu_{A^{m}}\left(s_{i}\right) \cdot \gamma_{k_{i}}$ is chosen as the parameter to be updated. $\psi_{k_{i}}\left(s_{i}\right)$ can be regard as the weight of the parameter vector.

3.2. Adaptive Scheme. Substituting (22) into (2), it can be expressed as

$$
\ddot{\mathbf{e}}+\frac{\mathbf{q}}{\mathbf{p}} \mathbf{K}^{-1} \dot{\mathbf{e}}^{2-\mathbf{p} / \mathbf{q}}+\mathbf{k}+\boldsymbol{\rho} \mathbf{s}=\boldsymbol{\varepsilon} .
$$

$\gamma_{k_{i}}^{*}$ is defined; then $k_{i}=\gamma_{k_{i}}^{* T} \Psi_{k_{i}}\left(s_{i}\right)$ is the optimal estimation for $\boldsymbol{\varepsilon}$. The optimal estimation error $w_{i}>0$ exists satisfying

$$
\left|\varepsilon_{i}-\gamma_{k_{i}}^{* T} \Psi_{k_{i}}\left(s_{i}\right)\right| \leq w_{i}
$$

Define

$$
\widetilde{\gamma}_{k_{i}}=\gamma_{k_{i}}-\gamma_{k_{i}}^{*}
$$

and then

$$
k_{i}=\widetilde{\gamma}_{k_{i}} \psi_{k_{i}}\left(s_{i}\right)+\gamma_{k_{i}}^{* T} \psi_{k_{i}}\left(s_{i}\right)
$$

The upper boundary of compensator is defined as $\sigma_{i}^{*}\left|s_{i}\right|$ satisfying

$$
w_{i} \leq \sigma_{i}^{*}\left|s_{i}\right|
$$

Define

$$
\tilde{\sigma}_{i}=\sigma_{i}-\sigma_{i}^{*}
$$

and then

$$
\rho_{i} s_{i}=\left(a_{i}+\sigma_{i}\right) s_{i}=a_{i} s_{i}+\sigma_{i} s_{i} .
$$


Choose the adaptive law as

$$
\begin{aligned}
\dot{\tilde{\gamma}}_{k_{i}} & =\frac{p_{i}}{q_{i}} K_{i} \dot{e}^{p_{i} / q_{i}-1} \eta_{\gamma_{k_{i}}} s_{i} \psi_{k_{i}}\left(s_{i}\right), \\
\dot{\tilde{\sigma}}_{i} & =\frac{p_{i}}{q_{i}} K_{i} \dot{e}^{p_{i} / q_{i}-1} \eta_{\sigma_{i}} s_{i}^{2},
\end{aligned}
$$

where $\eta_{{k_{i}}_{i}}$ and $\eta_{\sigma_{i}}$ are positive constants.

Choose the Lyapunov function as

$$
V=\frac{1}{2} \mathbf{s}^{T} \mathbf{s}+\frac{1}{2} \sum_{i=1}^{n}\left(\frac{1}{\eta_{\gamma_{k_{i}}}} \tilde{\gamma}_{k_{i}}^{T} \widetilde{\gamma}_{k_{i}}\right)+\frac{1}{2} \sum_{i=1}^{n}\left(\frac{1}{\eta_{\sigma_{i}}} \widetilde{\sigma}_{i}^{T} \widetilde{\sigma}_{i}\right) .
$$

Then, the derivative of $V$ can be obtained as

$$
\begin{aligned}
\dot{V}= & \mathbf{s}^{T} \dot{\mathbf{s}}+\sum_{i=1}^{n} \frac{1}{\eta_{\gamma_{k_{i}}}} \tilde{\gamma}_{k_{i}}^{T} \dot{\tilde{\gamma}}_{k_{i}}+\sum_{i=1}^{n} \frac{1}{\eta_{\sigma_{i}}} \widetilde{\sigma}_{i}^{T} \dot{\tilde{\sigma}}_{i} \\
= & \mathbf{s}^{T} \frac{p_{i}}{q_{i}} \mathbf{K} \dot{\mathbf{e}}^{p_{i} / q_{i}-1}(\boldsymbol{\varepsilon}-\mathbf{k}-\boldsymbol{\rho} \mathbf{s})+\sum_{i=1}^{n} \frac{1}{\eta_{\gamma_{k_{i}}}} \widetilde{\gamma}_{k_{i}}^{T} \dot{\tilde{\gamma}}_{k_{i}} \\
& +\sum_{i=1}^{n} \frac{1}{\eta_{\sigma_{i}}} \widetilde{\sigma}_{i}^{T} \dot{\tilde{\sigma}}_{i} \\
= & \sum_{i=1}^{n} s_{i} \frac{p_{i}}{q_{i}} K_{i} \dot{e}^{p_{i} / q_{i}-1}\left(\varepsilon_{i}-k_{i}-\rho_{i} s_{i}\right)+\sum_{i=1}^{n} \frac{1}{\eta_{\gamma_{k_{i}}}} \widetilde{\gamma}_{k_{i}}^{T} \dot{\tilde{\gamma}}_{k_{i}} \\
& +\sum_{i=1}^{n} \frac{1}{\eta_{\sigma_{i}}} \widetilde{\sigma}_{i}^{T} \dot{\tilde{\sigma}}_{i} .
\end{aligned}
$$

As $k_{i}=\widetilde{\gamma}_{k_{i}} \psi_{k_{i}}\left(s_{i}\right)+\gamma_{k_{i}}^{* T} \psi_{k_{i}}\left(s_{i}\right)$ and $\rho_{i} s_{i}=a_{i} s_{i}+\sigma_{i} s_{i}$, (36) can be expressed as

$$
\begin{aligned}
\dot{V} & =\sum_{i=1}^{n} s_{i} \frac{p_{i}}{q_{i}} K_{i} \dot{e}^{p_{i} / q_{i}-1}\left[\varepsilon_{i}-\widetilde{\gamma}_{k_{i}} \psi_{k_{i}}\left(s_{i}\right)-\gamma_{k_{i}}^{* T} \psi_{k_{i}}\left(s_{i}\right)\right. \\
& \left.-a_{i} s_{i}-\sigma_{i} s_{i}\right]+\sum_{i=1}^{n} \frac{1}{\eta_{f_{k_{i}}}} \widetilde{\gamma}_{k_{i}}^{T} \dot{\tilde{\gamma}}_{k_{i}}+\sum_{i=1}^{n} \frac{1}{\eta_{\sigma_{i}}} \widetilde{\sigma}_{i}^{T} \dot{\tilde{\sigma}}_{i}=\sum_{i=1}^{n} s_{i} \\
& . \frac{p_{i}}{q_{i}} K_{i} \dot{e}^{p_{i} / q_{i}-1}\left[\varepsilon_{i}-\widetilde{\gamma}_{k_{i}}^{T} \psi_{k_{i}}\left(s_{i}\right)-\gamma_{k_{i}}^{* T} \psi_{k_{i}}\left(s_{i}\right)-a_{i} s_{i}\right. \\
& \left.-\tilde{\sigma}_{i}^{T} s_{i}-\sigma_{i}^{*} s_{i}\right]+\sum_{i=1}^{n} \frac{1}{\eta_{\gamma_{k_{i}}}} \widetilde{\gamma}_{k_{i}}^{T} \dot{\tilde{\gamma}}_{k_{i}}+\sum_{i=1}^{n} \frac{1}{\eta_{\sigma_{i}}} \tilde{\sigma}_{i}^{T} \dot{\tilde{\sigma}}_{i} \\
& =-\sum_{i=1}^{n} \frac{p_{i}}{q_{i}} K_{i} \dot{e}^{p_{i} / q_{i}-1} s_{i} a_{i} s_{i}+\sum_{i=1}^{n} \frac{p_{i}}{q_{i}} K_{i} \dot{e}^{p_{i} / q_{i}-1} s_{i}\left[\varepsilon_{i}\right. \\
& \left.-\gamma_{k_{i}}^{* T} \psi_{k_{i}}\left(s_{i}\right)-\sigma_{i}^{*} s_{i}\right]+\left[\sum_{i=1}^{n} \frac{1}{\eta_{\gamma_{k_{i}}}} \widetilde{\gamma}_{k_{i}}^{T} \dot{\tilde{\gamma}}_{k_{i}}\right. \\
& \left.-\sum_{i=1}^{n} \frac{p_{i}}{q_{i}} K_{i} \dot{e}^{p_{i} / q_{i}-1} s_{i} \widetilde{\gamma}_{k_{i}}^{T} \psi_{k_{i}}\left(s_{i}\right)\right]+\left[\sum_{i=1}^{n} \frac{1}{\eta_{\sigma_{i}}} \widetilde{\sigma}_{i}^{T} \dot{\tilde{\sigma}}_{i}\right.
\end{aligned}
$$

$$
\begin{aligned}
& \left.-\sum_{i=1}^{n} \frac{p_{i}}{q_{i}} K_{i} \dot{e}^{p_{i} / q_{i}-1} s_{i} \tilde{\sigma}_{i}^{T} s_{i}\right]=-\sum_{i=1}^{n} \frac{p_{i}}{q_{i}} K_{i} \dot{e}^{p_{i} / q_{i}-1} s_{i} a_{i} s_{i} \\
& +\sum_{i=1}^{n} \frac{p_{i}}{q_{i}} K_{i} \dot{e}^{p_{i} / q_{i}-1} s_{i}\left[\varepsilon_{i}-\gamma_{k_{i}}^{* T} \psi_{k_{i}}\left(s_{i}\right)-\sigma_{i}^{*} s_{i}\right] \\
& +\sum_{i=1}^{n} \widetilde{\gamma}_{k_{i}}^{T}\left[\frac{1}{\eta_{\gamma_{i}}} \dot{\tilde{\gamma}}_{k_{i}}-\frac{p_{i}}{q_{i}} K_{i} \dot{e}^{p_{i} / q_{i}-1} s_{i} \psi_{k_{i}}\left(s_{i}\right)\right] \\
& +\sum_{i=1}^{n} \widetilde{\sigma}_{i}^{T}\left[\frac{1}{\eta_{\sigma_{i}}} \dot{\tilde{\sigma}}_{i}-\frac{p_{i}}{q_{i}} K_{i} \dot{e}^{p_{i} / q_{i}-1} s_{i}^{2}\right] .
\end{aligned}
$$

Since the adaptive law is $\dot{\tilde{\gamma}}_{k_{i}}=\left(p_{i} / q_{i}\right) K_{i} \dot{e}^{p_{i} / q_{i}-1} \eta_{\gamma_{k_{i}}} s_{i} \psi_{k_{i}}\left(s_{i}\right)$ and $\dot{\tilde{\sigma}}_{i}=\left(p_{i} / q_{i}\right) K_{i} e^{p_{i} / q_{i}-1} \eta_{\sigma_{i}} s_{i}^{2}$, then

$$
\begin{aligned}
\dot{V}= & -\sum_{i=1}^{n} \frac{p_{i}}{q_{i}} K_{i} \dot{e}^{p_{i} / q_{i}-1} s_{i} a_{i} s_{i} \\
& +\sum_{i=1}^{n} \frac{p_{i}}{q_{i}} K_{i} \dot{e}^{p_{i} / q_{i}-1} s_{i}\left[\varepsilon_{i}-\gamma_{k_{i}}^{* T} \psi_{k_{i}}\left(s_{i}\right)-\sigma_{i}^{*} s_{i}\right] .
\end{aligned}
$$

Since $\left|\varepsilon_{i}-\gamma_{k_{i}}^{* T} \Psi_{k_{i}}\left(s_{i}\right)\right| \leq w_{i} \leq \sigma_{k_{i}}^{*}\left|s_{i}\right|$, then

$$
\begin{aligned}
& \frac{p_{i}}{q_{i}} K_{i} \dot{e}^{p_{i} / q_{i}-1} s_{i}\left[\varepsilon_{i}-\gamma_{k_{i}}^{* T} \psi_{k_{i}}\left(s_{i}\right)\right] \\
& \quad \leq \frac{p_{i}}{q_{i}} K_{i} \dot{e}^{p_{i} / q_{i}-1}\left|s_{i}\right|\left|\varepsilon_{i}-\gamma_{k_{i}}^{* T} \psi_{k_{i}}\left(s_{i}\right)\right| \\
& \quad \leq \frac{p_{i}}{q_{i}} K_{i} \dot{e}^{p_{i} / q_{i}-1}\left|s_{i}\right| w_{i} \leq \frac{p_{i}}{q_{i}} K_{i} \dot{e}^{p_{i} / q_{i}-1} \sigma_{i}^{*} s_{i}^{2} .
\end{aligned}
$$

Then (39) becomes

$$
\begin{aligned}
\dot{V} \leq & -\sum_{i=1}^{n} \frac{p_{i}}{q_{i}} K_{i} \dot{e}^{p_{i} / q_{i}-1} s_{i} a_{i} s_{i} \\
& +\sum_{i=1}^{n} \frac{p_{i}}{q_{i}} K_{i} \dot{e}^{p_{i} / q_{i}-1}\left(\left|s_{i}\right| w_{i}-\sigma_{i}^{*} s_{i}^{2}\right) \\
\leq & -\sum_{i=1}^{n} \frac{p_{i}}{q_{i}} K_{i} \dot{e}^{p_{i} / q_{i}-1} s_{i} a_{i} s_{i} .
\end{aligned}
$$

Since $a_{i}$ is a positive constant, therefore $\dot{V}<0$ when $\dot{e} \neq 0$ and $s \neq 0$.

For $\dot{e}=0$, it is obtained as (41) by substituting (22) into (2).

$$
\ddot{\mathbf{e}}=-\mathbf{k}-\boldsymbol{\rho} \mathbf{s}+\boldsymbol{\varepsilon} \neq 0 .
$$

Therefore, $\dot{e}=0$ is not an attractor in the reaching phase, and $\dot{V}=0$ only when $s=0$. Thus, it is proved that the adaptive law in (34) drives the tracking error to converge to zero in finite time. Therefore, the actual trajectory of the manipulator end-effector $\mathbf{x}$ converges to the desired trajectory $\mathbf{x}_{\mathbf{d}}$ in the task space. 


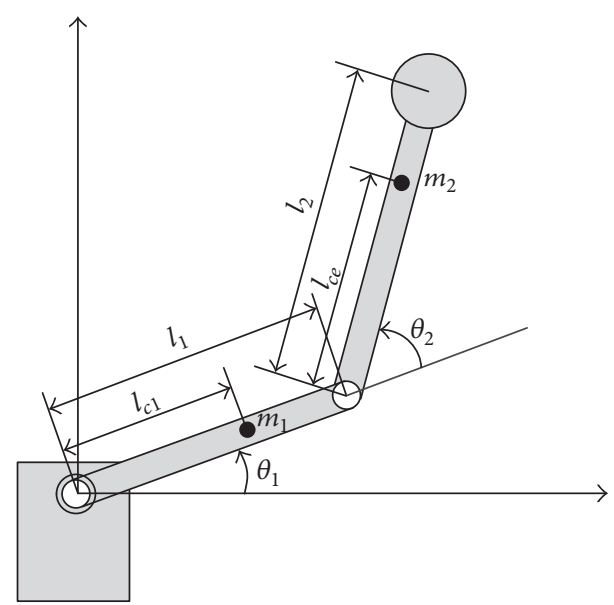

FIGURE 2: Schematic diagram of the 2-DOF robot manipulator.

\section{Simulation}

4.1. Simulation Setup. In order to verify the effectiveness of the proposed controller by simulation, a 2-DOF robot manipulator is adopted, which is shown in Figure 2.

The Jacobian matrix of this manipulator is shown as

$$
\begin{aligned}
& \mathbf{J}(\boldsymbol{\theta}) \\
& =\left[\begin{array}{ll}
-l_{1} \sin \left(\theta_{1}\right)-l_{2} \sin \left(\theta_{1}+\theta_{2}\right) & -l_{2} \sin \left(\theta_{1}+\theta_{2}\right) \\
l_{1} \cos \left(\theta_{1}\right)+l_{2} \cos \left(\theta_{1}+\theta_{2}\right) & l_{2} \cos \left(\theta_{1}+\theta_{2}\right)
\end{array}\right],
\end{aligned}
$$

where $l_{1}$ and $l_{2}$ denote the length of first link and second link, respectively; $\theta_{1}$ and $\theta_{2}$ are the joint position of the two links.

The dynamic model of the robot manipulator system is given for simulation. The dynamic model is given as (1). Details of the model are shown as

$$
\begin{aligned}
\mathbf{M}(\boldsymbol{\theta}) & =\left[\begin{array}{cc}
\alpha+2 \delta \cos \left(\theta_{2}\right)+2 \eta \sin \left(\theta_{2}\right) & \beta+\delta \cos \left(\theta_{2}\right)+\eta \sin \left(\theta_{2}\right) \\
\beta+\delta \cos \left(\theta_{2}\right)+\eta \sin \left(\theta_{2}\right) & \beta
\end{array}\right], \\
\mathbf{C}(\boldsymbol{\theta}, \dot{\boldsymbol{\theta}}) & =\left[\begin{array}{cc}
{\left[-2 \delta \sin \left(\theta_{2}\right)+2 \eta \cos \left(\theta_{2}\right)\right] \dot{\theta}_{2}} & {\left[-\delta \sin \left(\theta_{2}\right)+\eta \cos \left(\theta_{2}\right)\right] \dot{\theta}_{2}} \\
{\left[\delta \sin \left(\theta_{2}\right)-\eta \cos \left(\theta_{2}\right)\right] \dot{\theta}_{1}} & 0
\end{array}\right], \\
\mathbf{G}(\boldsymbol{\theta}) & =\left[\begin{array}{c}
\delta e_{2} \cos \left(\theta_{1}+\theta_{2}\right)+\eta e_{2} \sin \left(\theta_{1}+\theta_{2}\right)+\left(\alpha-\beta+e_{1}\right) e_{2} \cos \left(\theta_{1}\right) \\
\delta e_{2} \cos \left(\theta_{1}+\theta_{2}\right)+\eta e_{2} \sin \left(\theta_{1}+\theta_{2}\right)
\end{array}\right] .
\end{aligned}
$$

The friction term affects the control performance of robot system seriously. Thus, the friction term is chosen as

$$
\mathbf{F}(\boldsymbol{\theta}, \dot{\boldsymbol{\theta}})=\left[\begin{array}{l}
F_{v 1} \dot{\theta}_{1}+F_{c 1} \operatorname{sgn}\left(\dot{\theta}_{1}\right) \\
F_{v 2} \dot{\theta}_{2}+F_{c 2} \operatorname{sgn}\left(\dot{\theta}_{2}\right)
\end{array}\right],
$$

where $\alpha=I_{1}+m_{1} l_{c 1}^{2}+I_{e}+m_{e} l_{c e}^{2}+m_{e} l_{1}^{2}, \beta=I_{e}+m_{e} l_{c e}^{2}$, $\delta=m_{e} l_{1} l_{c e} \cos \delta_{e}, \eta=m_{e} l_{1} l_{c e} \sin \delta_{e}, e_{1}=m_{1} l_{1} l_{c 1}-I_{1}-m_{1} l_{1}^{2}$, $e_{2}=g / l_{1} ; m_{1}$ denotes the mass of first link; $l_{c 1}$ is the distance between the mass center of first link and the first joint; $I_{1}$ is the moment of inertia of the first link; $m_{e}$ is the mass of second link with payload; $l_{c e}$ is the distance between the mass center of second link and the second joint; $I_{e}$ is the moment of inertia of the second link; $\delta_{e}$ is the angle relative to the original second link. The physical parameters of the robot manipulator are shown in Table 1.

The desired trajectory of end-effector in the task space is designed as a circle, which is expressed as

$$
\mathbf{x}_{\mathbf{d}}=\left[\begin{array}{l}
1+0.6 \cos \left(\frac{\pi t}{5}\right) \\
1+0.6 \sin \left(\frac{\pi t}{5}\right)
\end{array}\right] \text {. }
$$

Three controllers are chosen to control the manipulator plant. Controller 1 is conventional TDE based NTSM control method. According to the study of Jin et al. [24], controller 1 is expressed as

$$
\begin{aligned}
\boldsymbol{\tau}= & \boldsymbol{\tau}_{t-L}-\overline{\mathbf{M}}_{t-L} \\
& +\overline{\mathbf{M}}\left[\ddot{\boldsymbol{\theta}}_{d}+\frac{\mathbf{q}}{\mathbf{p}} \mathbf{K}^{-1} \dot{\mathbf{e}}_{\boldsymbol{\theta}}^{2-\mathbf{p} / \mathbf{q}}+\mathbf{K}_{\mathbf{s w}} \operatorname{sat}(\mathbf{s}, \boldsymbol{\Phi})\right],
\end{aligned}
$$

where

$$
\{\operatorname{sat}(s, \Phi)\}_{i}= \begin{cases}\frac{s_{i}}{\left|s_{i}\right|} & \text { if }\left|s_{i}\right| \geq \Phi_{i} \\ \frac{s_{i}}{\Phi_{i}} & \text { if }\left|s_{i}\right| \leq \Phi_{i} .\end{cases}
$$

and it is the replacement function of sign function to reduce the chattering; $\mathbf{e}_{\boldsymbol{\theta}}$ is the tracking error and $\mathbf{e}_{\boldsymbol{\theta}}=\boldsymbol{\theta}_{\mathbf{d}}-\boldsymbol{\theta}$. Controller 2 is the improved controller based on controller 1 , which is expressed as (15). Similarly, to reduce the chattering, the sign function existing in controller is replaced by saturation function. Controller 3 is the proposed controller, which is expressed as (22).

After the tuning process, the gains of controller 1 are $\overline{\mathbf{M}}=$ $0.15 \mathbf{I}, \mathbf{K}=0.9 \mathbf{I}, \mathbf{p}=5 \mathbf{I}, \mathbf{q}=3 \mathbf{I}, \mathbf{K}_{\mathrm{sw}}=10 \mathbf{I}$, and $\boldsymbol{\Phi}=0.04 \mathbf{I}$. The gains of controller 2 are $\overline{\mathbf{M}}=0.15 \mathbf{I}, \mathbf{K}=0.9 \mathbf{I}, \mathbf{p}=5 \mathbf{I}$, $\mathbf{q}=3 \mathbf{I}, \mathbf{K}_{\text {sw }}=10 \mathbf{I}$, and $\boldsymbol{\Phi}=0.04 \mathbf{I}$. The gains of proposed controller are $\overline{\mathbf{M}}=0.15 \mathbf{I}, \mathbf{K}=0.9 \mathbf{I}, \mathbf{p}=5 \mathbf{I}, \mathbf{q}=3 \mathbf{I}, \eta_{\theta_{k_{i}}}=10$, and $\eta_{\sigma_{i}}=200 ; a_{i}=200$. 
TABLE 1: The physical parameters of the robot manipulator.

\begin{tabular}{ccccccccccccc}
\hline$l_{1}$ & $l_{2}$ & $l_{c 1}$ & $l_{c e}$ & $I_{1}$ & $I_{2}$ & $m_{1}$ & $m_{e}$ & $\delta_{e}$ & $F_{v 1}$ & $F_{c 1}$ & $F_{v 2}$ & $F_{c 2}$ \\
\hline 1 & 1.2 & 0.5 & 1 & 0.083 & 0.4 & 1 & 3 & 0 & 10 & 10 & 10 & 10 \\
\hline
\end{tabular}

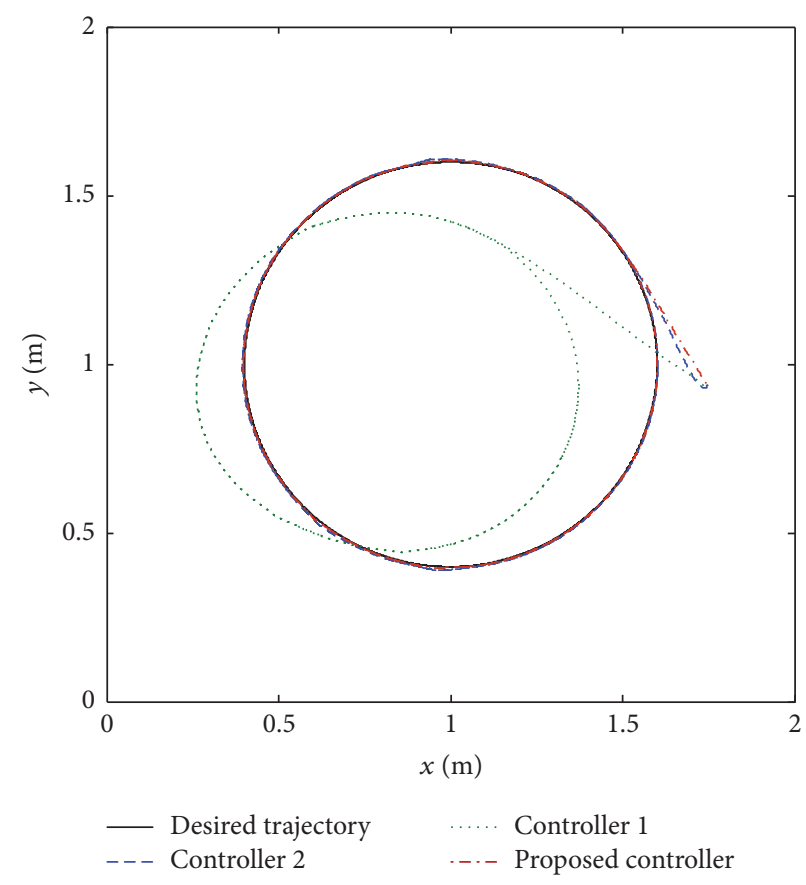

Figure 3: The desired circle trajectory with kinematic uncertainties.

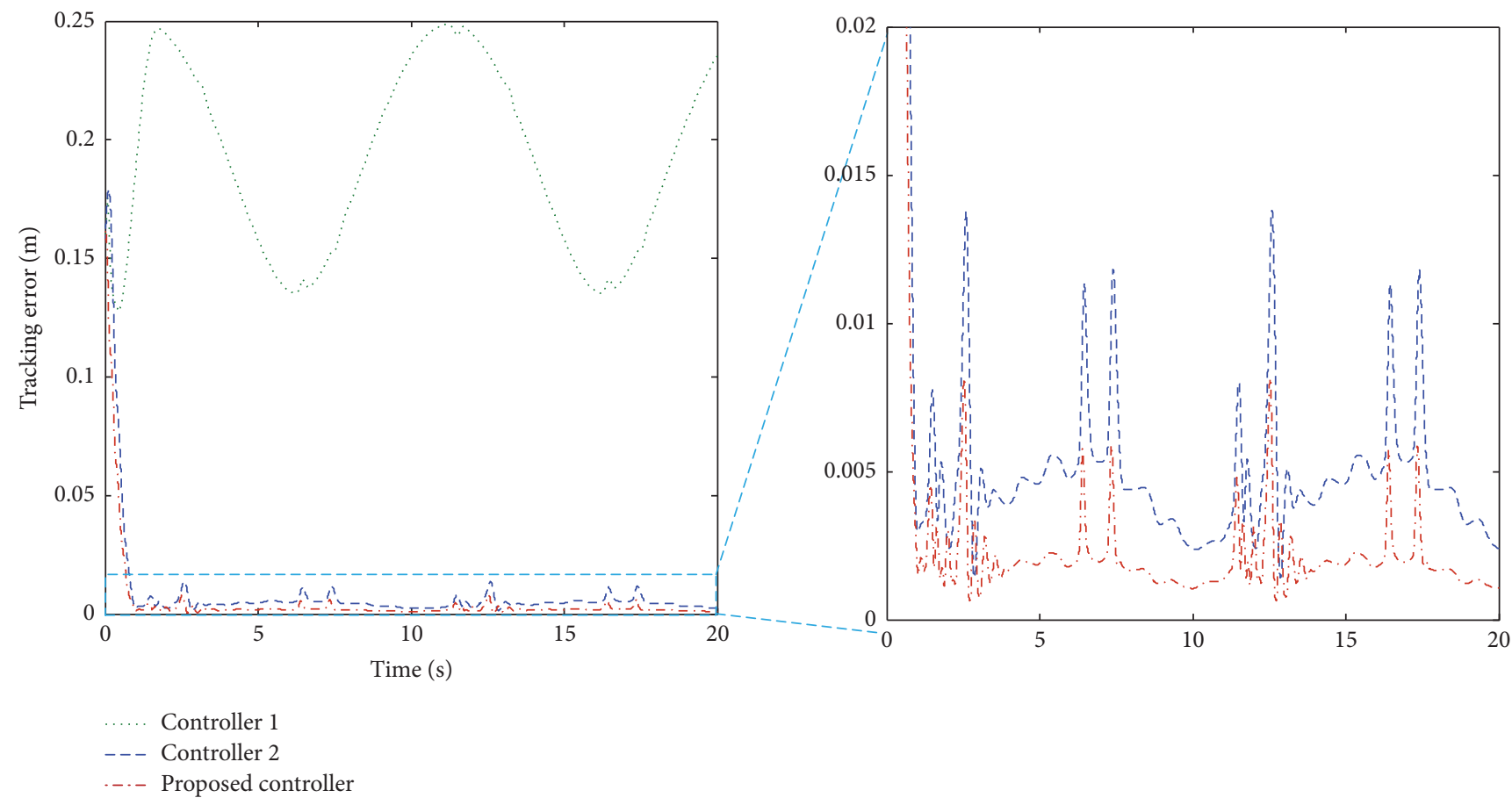

FIGURE 4: Tracking error of manipulator end-effector with kinematic uncertainties. 

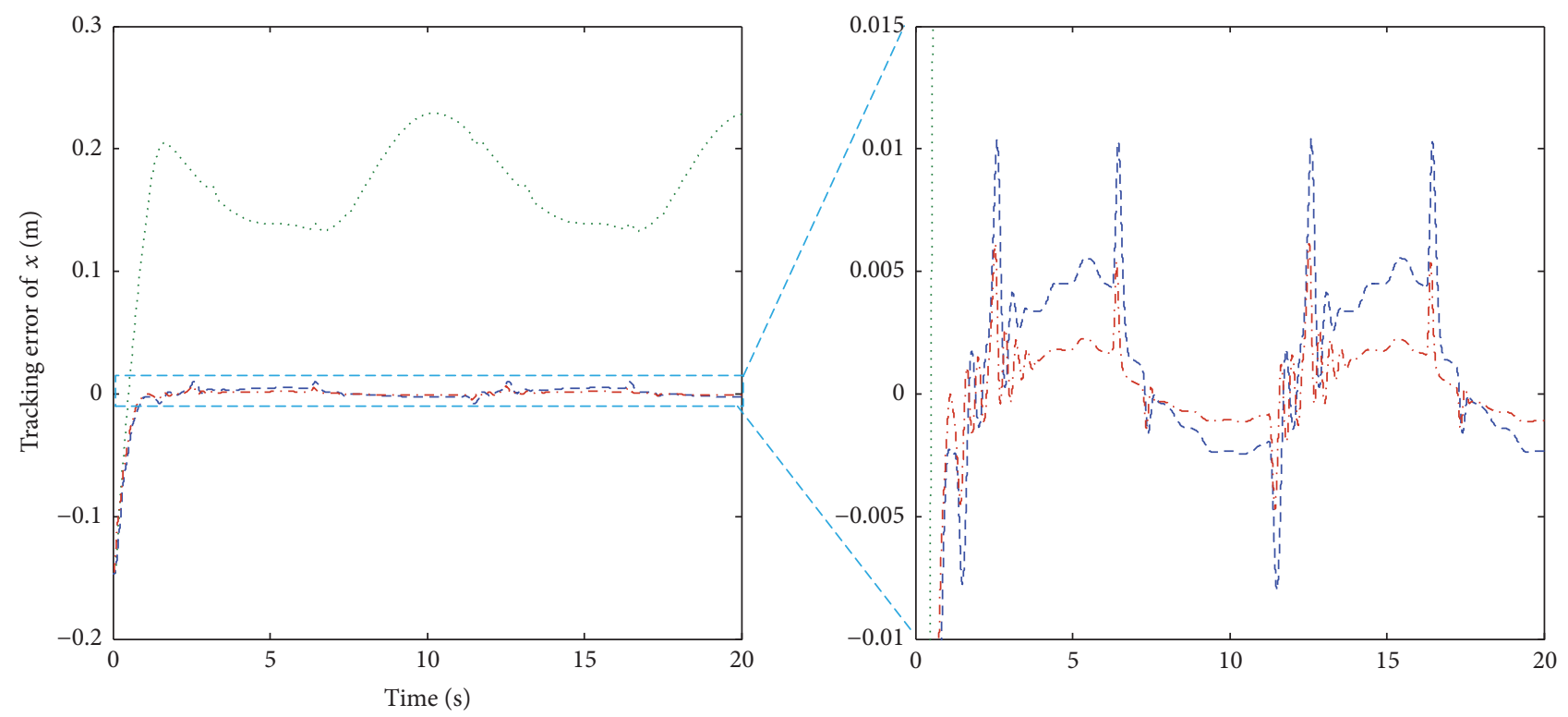

.... Controller 1

- - - Controller 2

-..- Proposed controller

(a)


Controller 1

- - Controller 2

-..- Proposed controller

(b)

FIGURE 5: Tracking error of $x$ and $y$ directions with kinematic uncertainties.

Three controllers are designed based on TDE. They are easy to implement and no information about the dynamic model is required. Because kinematics uncertainties are considered in controllers 2 and 3 , the values of $l_{1}$ and $l_{2}$ are required. In this simulation, it is assumed that the values of $l_{1}$ and $l_{2}$ are not exactly known, and they are estimated as $l_{1}=1.2, l_{2}=1.3$. The other parameters of the robot manipulator are not required.

The simulation is carried out by three cases. Case 1 is the simulation with the external kinematics uncertainties. Case 2 is the simulation with external dynamic uncertainties. Case 3 is the simulation with measurement noise. 


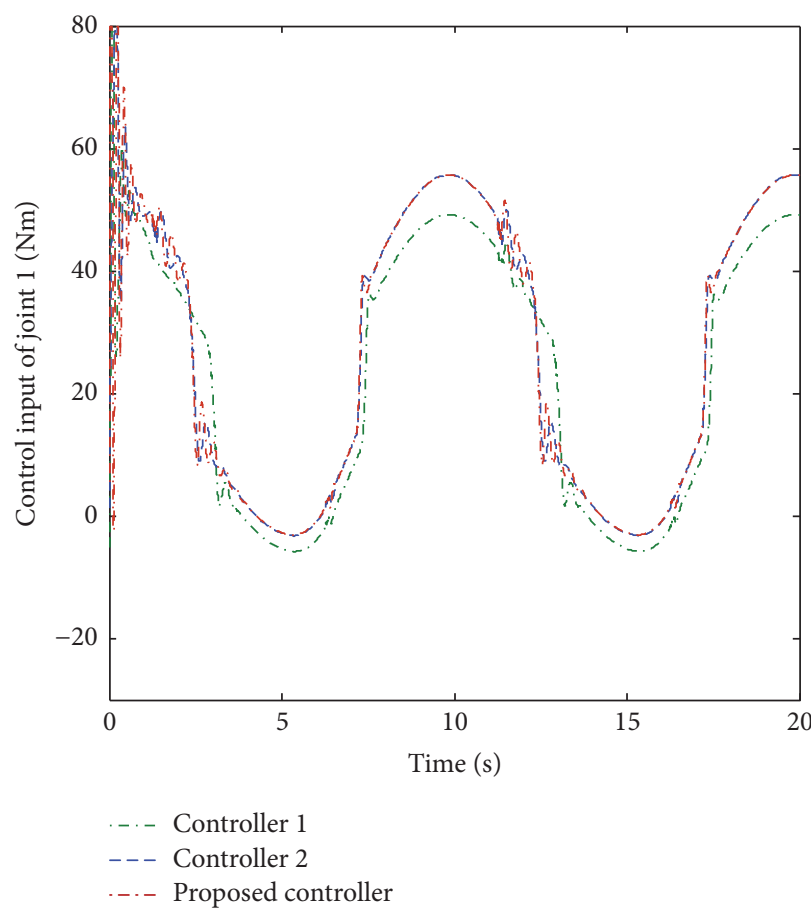

(a)

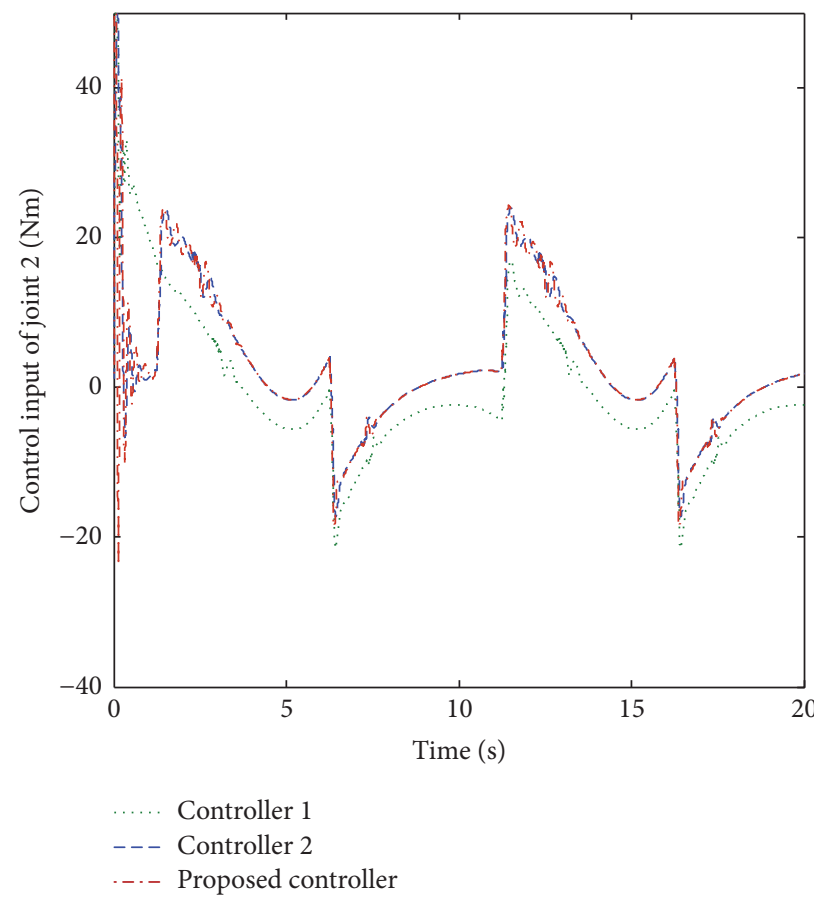

(b)

FIgURE 6: Control input of joint 1 and joint 2 with kinematic uncertainties.

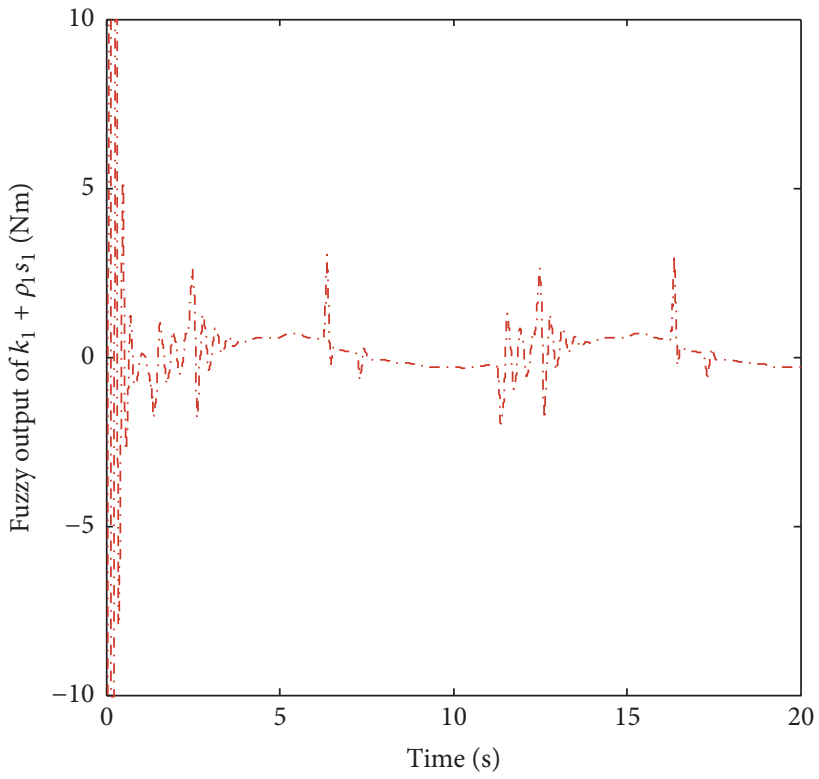

(a)



(b)

FIgURE 7: Fuzzy output of $\mathbf{k}+\boldsymbol{\rho}$ s with kinematic uncertainties.

\subsection{Results and Discussion}

4.2.1. Case 1 with External Kinematic Uncertainties. In this case, the simulation is carried out on the plant model with kinematic uncertainties. It is assumed that the size of the two links varies as $l_{1}=1.2+0.1 \sin (2 \pi t), l_{2}=1.3+0.1 \sin (2 \pi t)$.
Three controllers are applied to control the plant, respectively. The results are shown in Figures 3-7. Maximum values (MAX) and root mean square (RMS) of tracking errors are shown in Figures 8 and 9. MAX and RMS are calculated by the simulation data in the second period. 


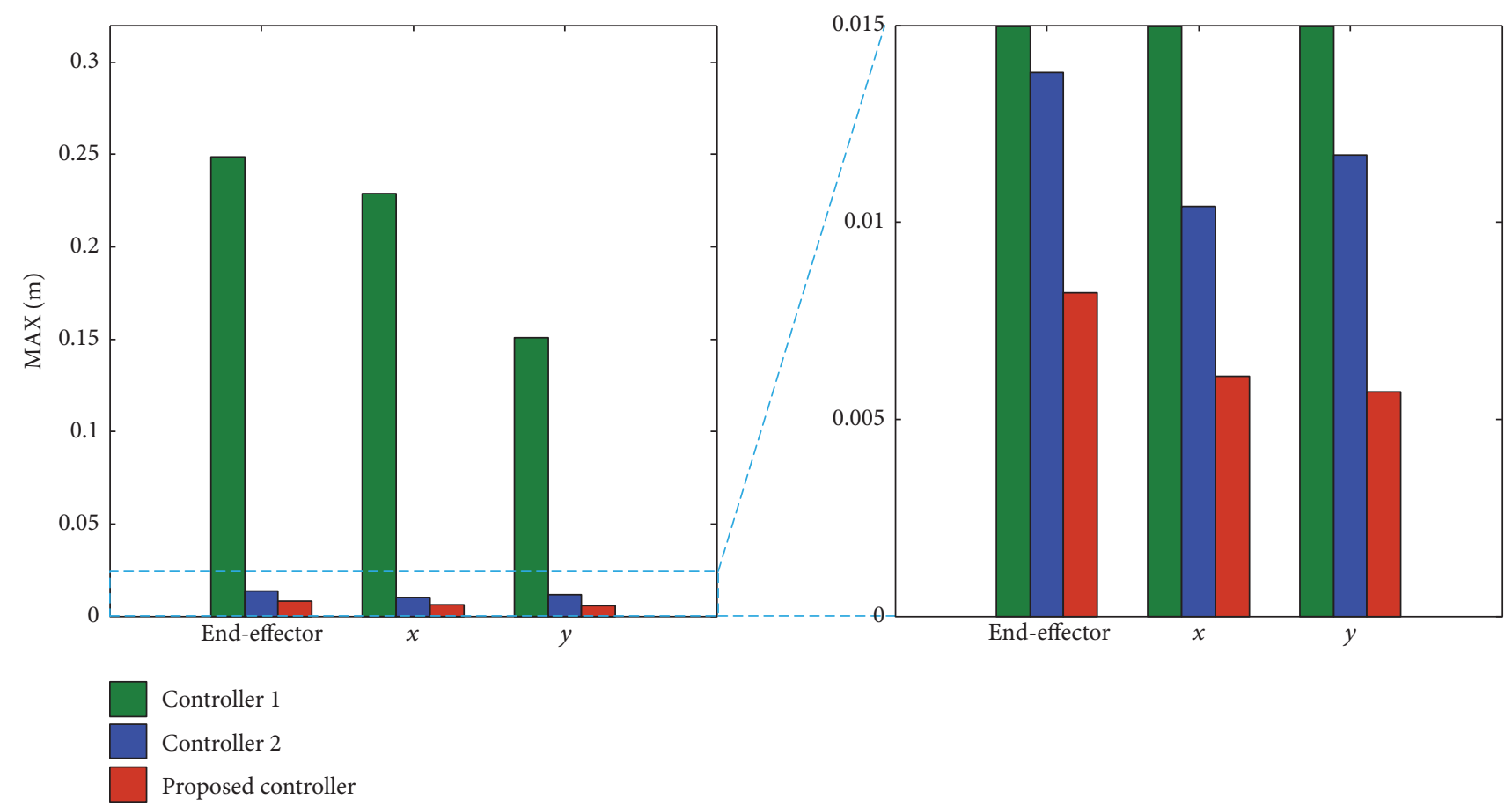

FIGURE 8: MAX of tracking errors with kinematic uncertainties.

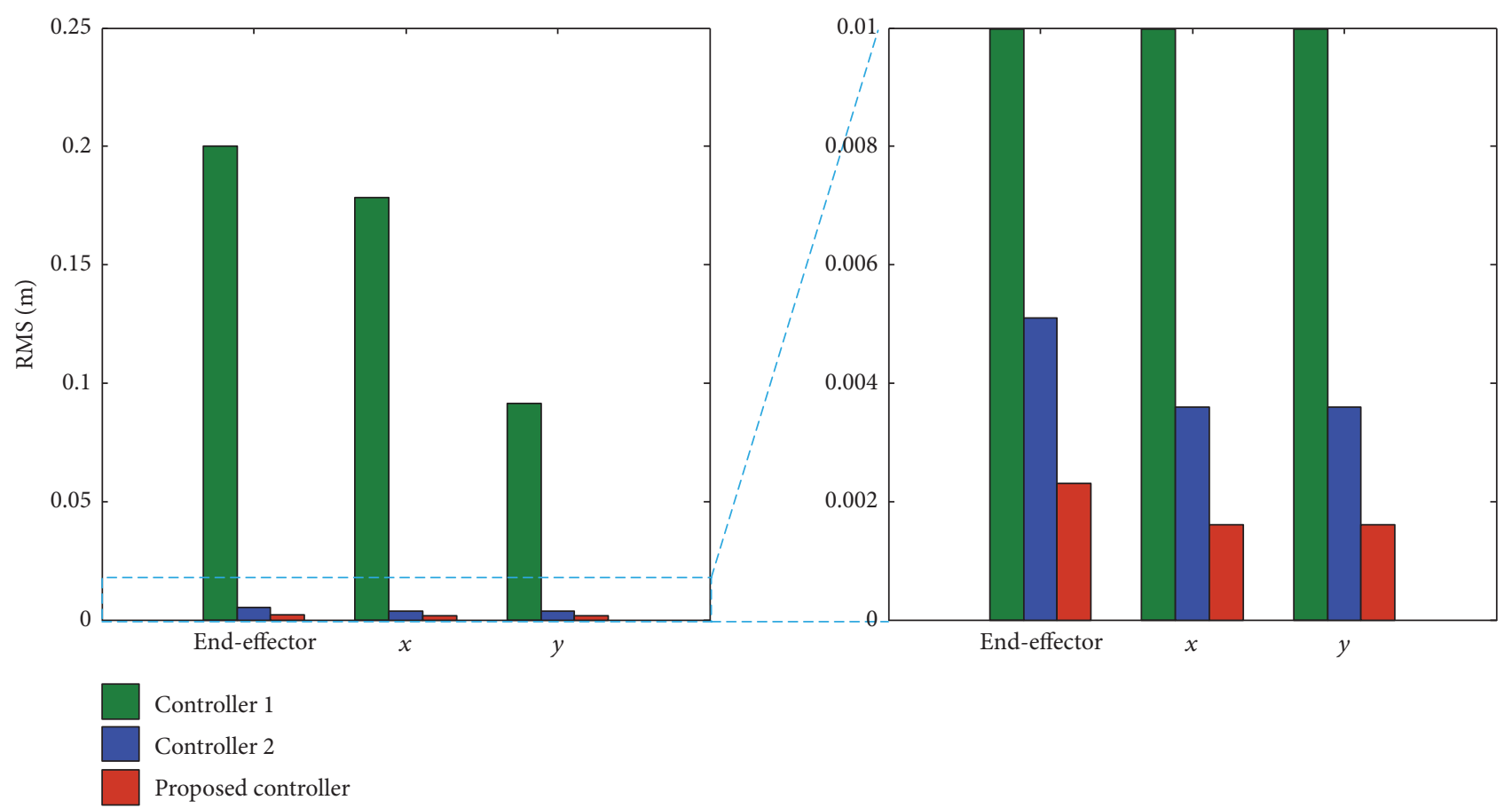

FIGURE 9: RMS of tracking errors with kinematic uncertainties.

From Figures 3-7, it can be observed that the desired trajectory can be tracked well under controller 2 and the proposed controller, while the trajectory can not be tracked well under controller 1 . The reason is that controller 1 does not consider the kinematic uncertainties, and the kinematic parameters are estimated. When errors exist between real size and estimation size of the link, the large tracking errors occur in the trajectory of the manipulator end-effector in the task space. From Figures 4 and 5, it is observed that peaks of tracking error exist during the control procedure of 


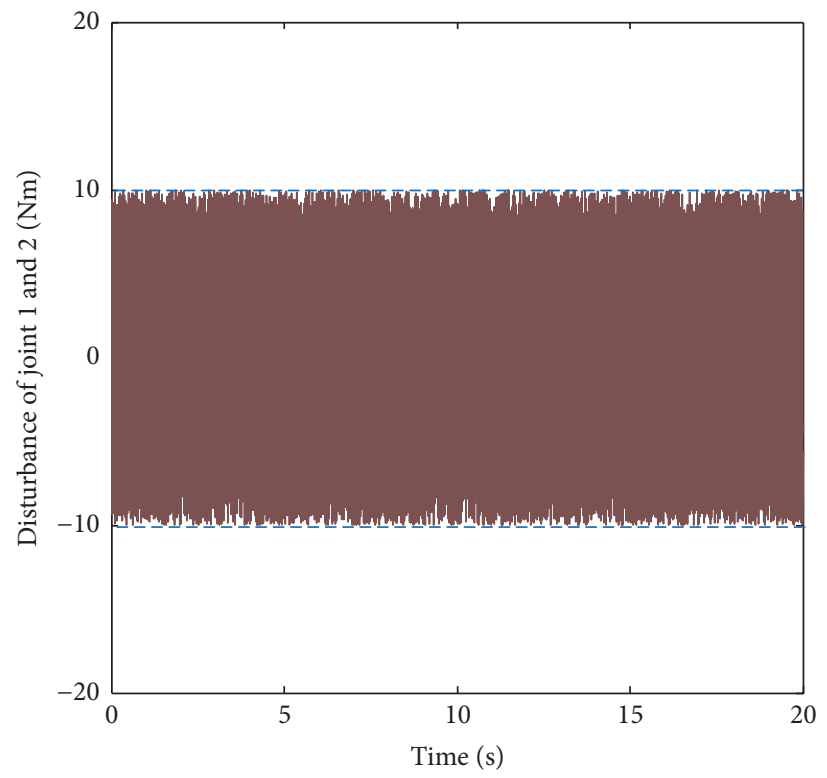

FIGURE 10: The external dynamic uncertainties.

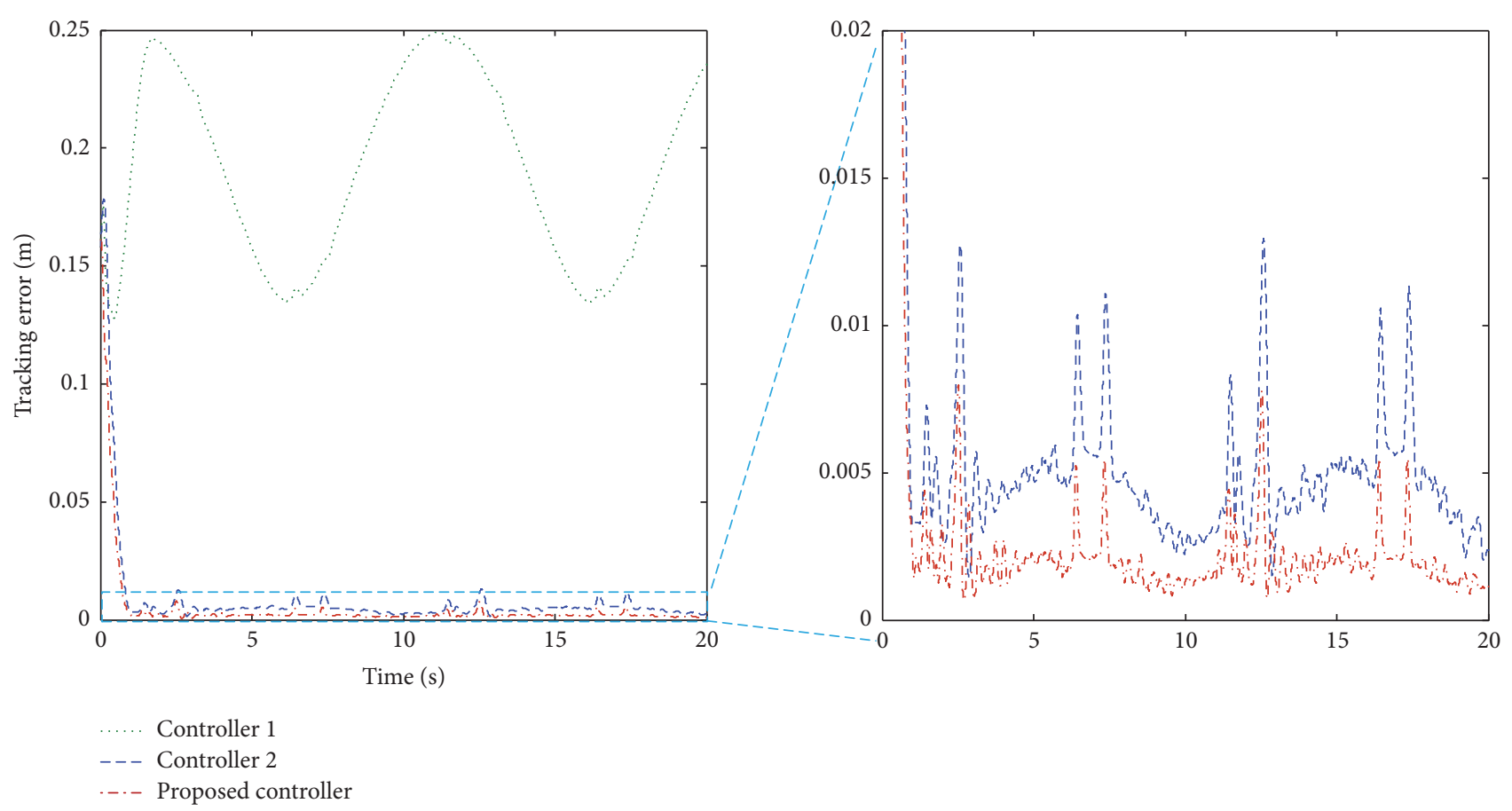

FIGURE 11: Tracking error of manipulator end-effector with dynamic uncertainties.

controller 2 and proposed controller. Those peaks are mainly caused by TDE error, due to Coulomb friction. It can also be observed that the peaks under the proposed controller are the smallest, which benefited from the compensation by adaptive fuzzy logic control scheme. The corresponding results are also shown in Figure 7. The fuzzy logic scheme output of proposed control provides relative larger control signal when the error peaks occur, which is essential for obtaining better control performance under large errors. It is obvious that the proposed controller has the highest tracking precision and fastest convergence rate among the three controllers, and the conventional TDE based NTSM controller has the relative worst performance. As shown in Figures 8 and 9, the tracking error of proposed controller has the smallest MAX under kinematic uncertainties. The MAX of proposed controller is $3.2 \%$ and $59.4 \%$ of those from the other two controllers. 

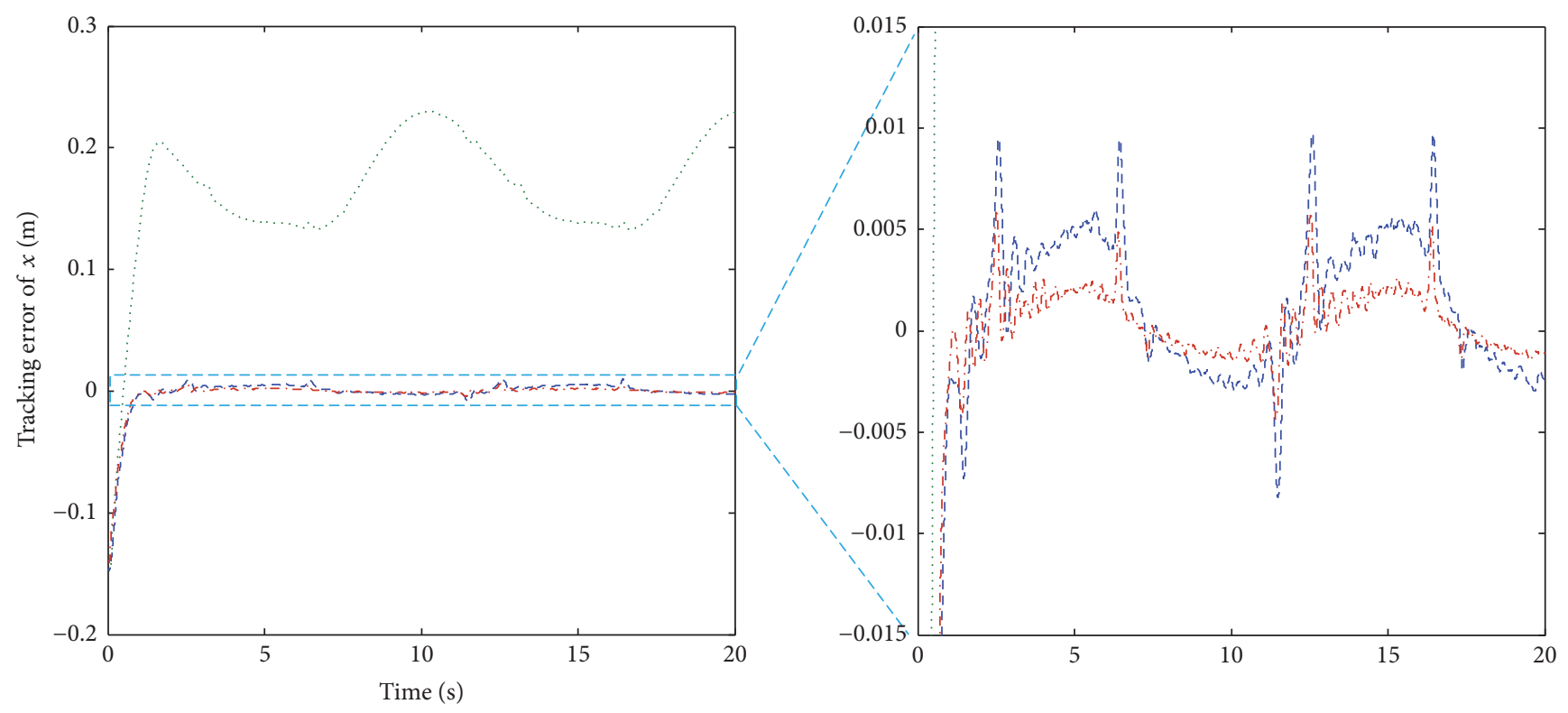

... Controller 1

- - - Controller 2

...- Proposed controller

(a)
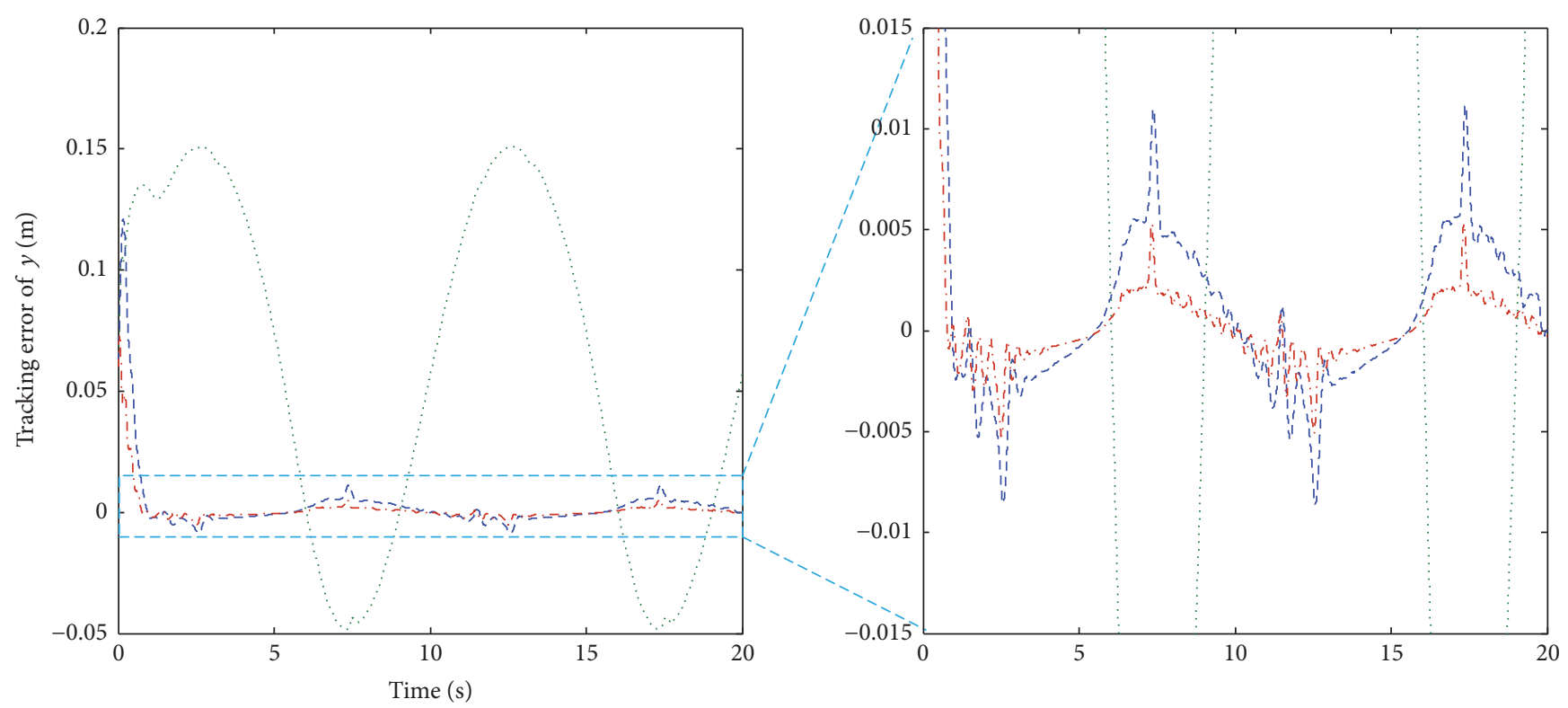

Controller 1

- - - Controller 2

...- Proposed controller

(b)

Figure 12: Tracking error of $x$ and $y$ directions with dynamic uncertainties.

The RMS of proposed controller is $1.1 \%$ and $45.1 \%$ of those from the other two controllers. From the above results, best performance is got by the proposed controller under kinematic uncertainties in the task space. Furthermore, no chattering is observed using those three controllers.
4.2.2. Case 2 with External Dynamic Uncertainties. In this case, the simulation is carried out on the plant model with dynamic uncertainties. The external dynamic uncertainties are shown in Figure 10. Three controllers are applied to control the plant, respectively. The results are shown in 


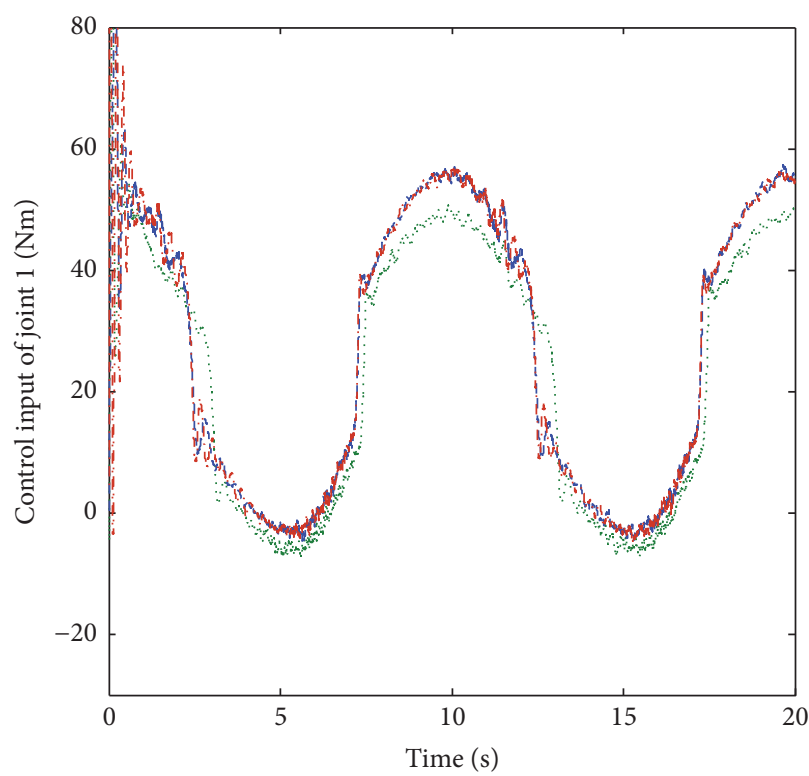

Controller 1

- - - Controller 2

-..- Proposed controller

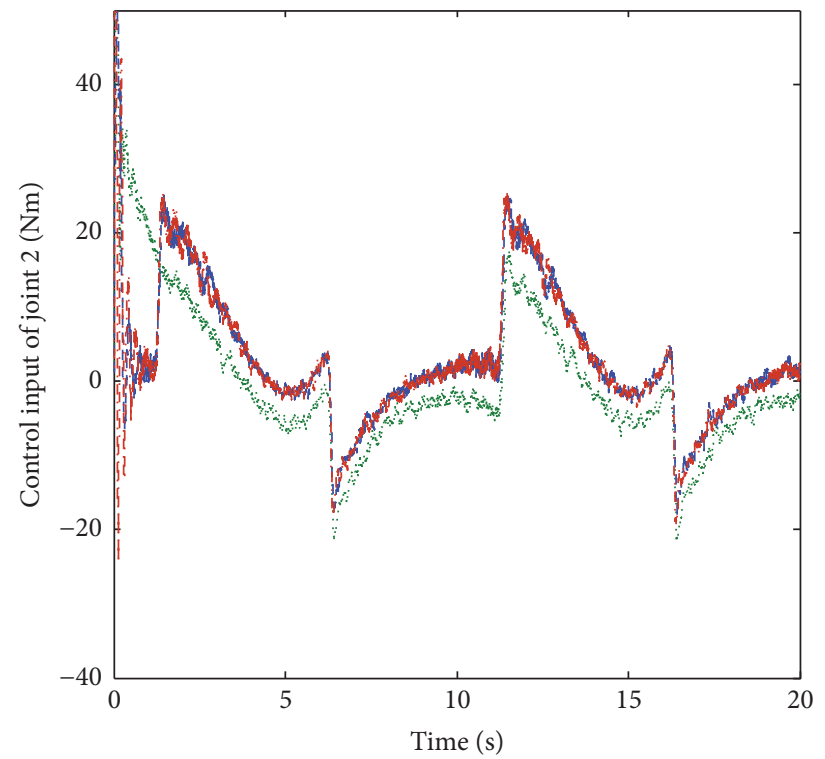

Controller 1

- - - Controller 2

...- Proposed controller

(a)

(b)

FIgURE 13: Control input of joint 1 and joint 2 with dynamic uncertainties.

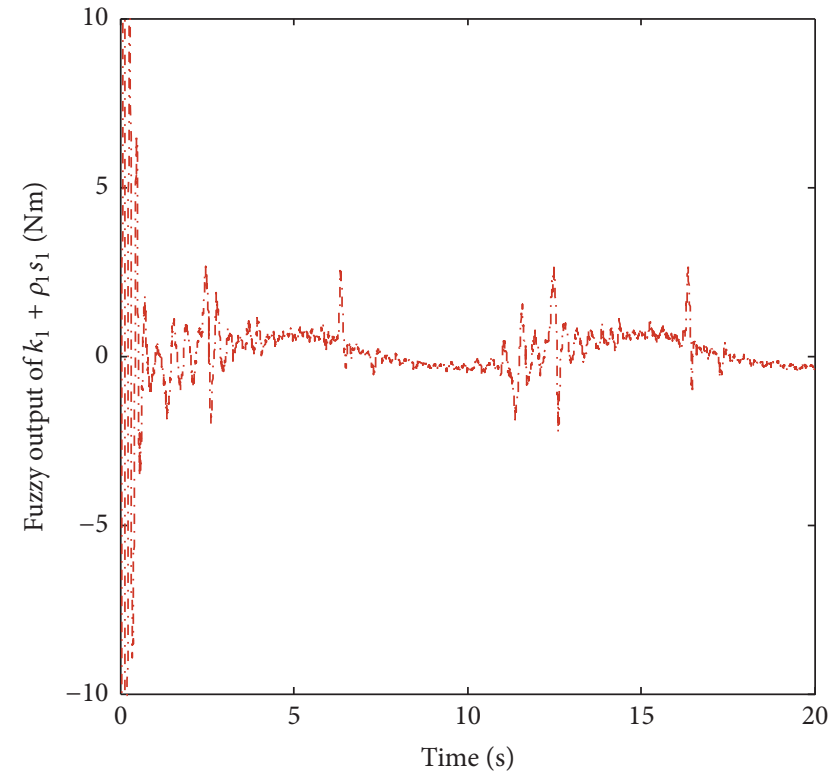

(a)

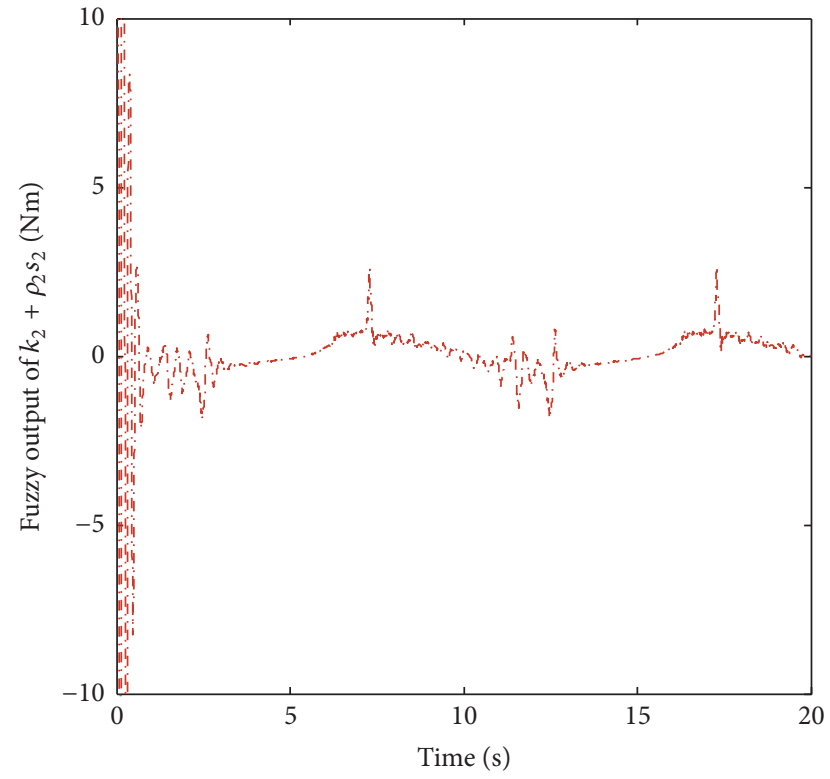

(b)

FIGURE 14: Fuzzy output of $\mathbf{k}+\rho \mathbf{s}$ with dynamic uncertainties.

Figures 11-14. MAX and RMS of tracking errors are shown in Figures 15 and 16. MAX and RMS are calculated by the experiment data in the second period.

It can be observed that controller 1 has a relative large tracking error, which is caused by the estimation of $l_{1}$ and $l_{2}$. Controller 2 and the proposed controller can track the desired trajectory with smaller error under external dynamic uncertainties, which verifies the robustness of the proposed controller. It can also be observed that the proposed controller has the highest tracking precision and fastest 


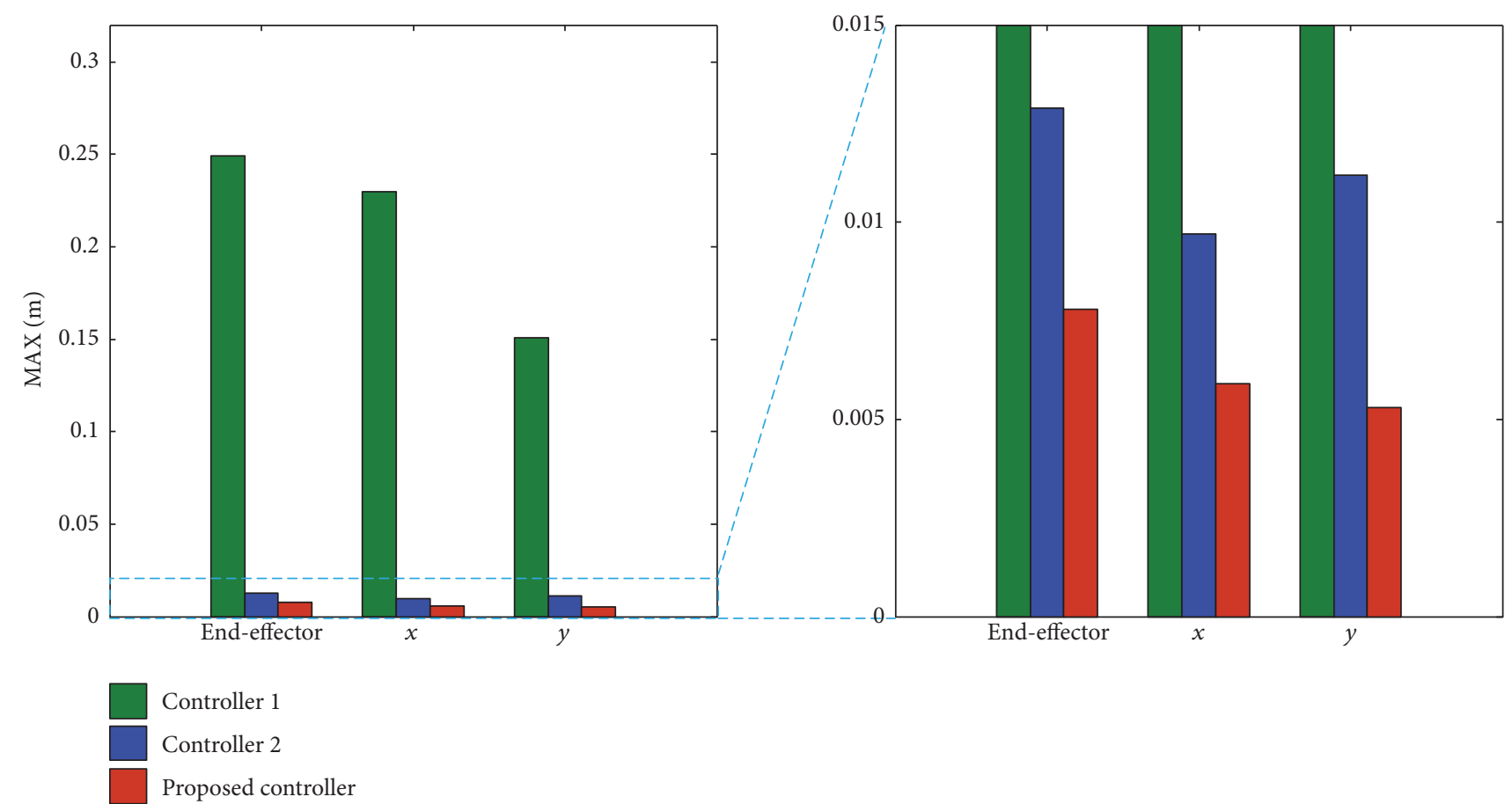

FIGURE 15: MAX of tracking errors with dynamic uncertainties.

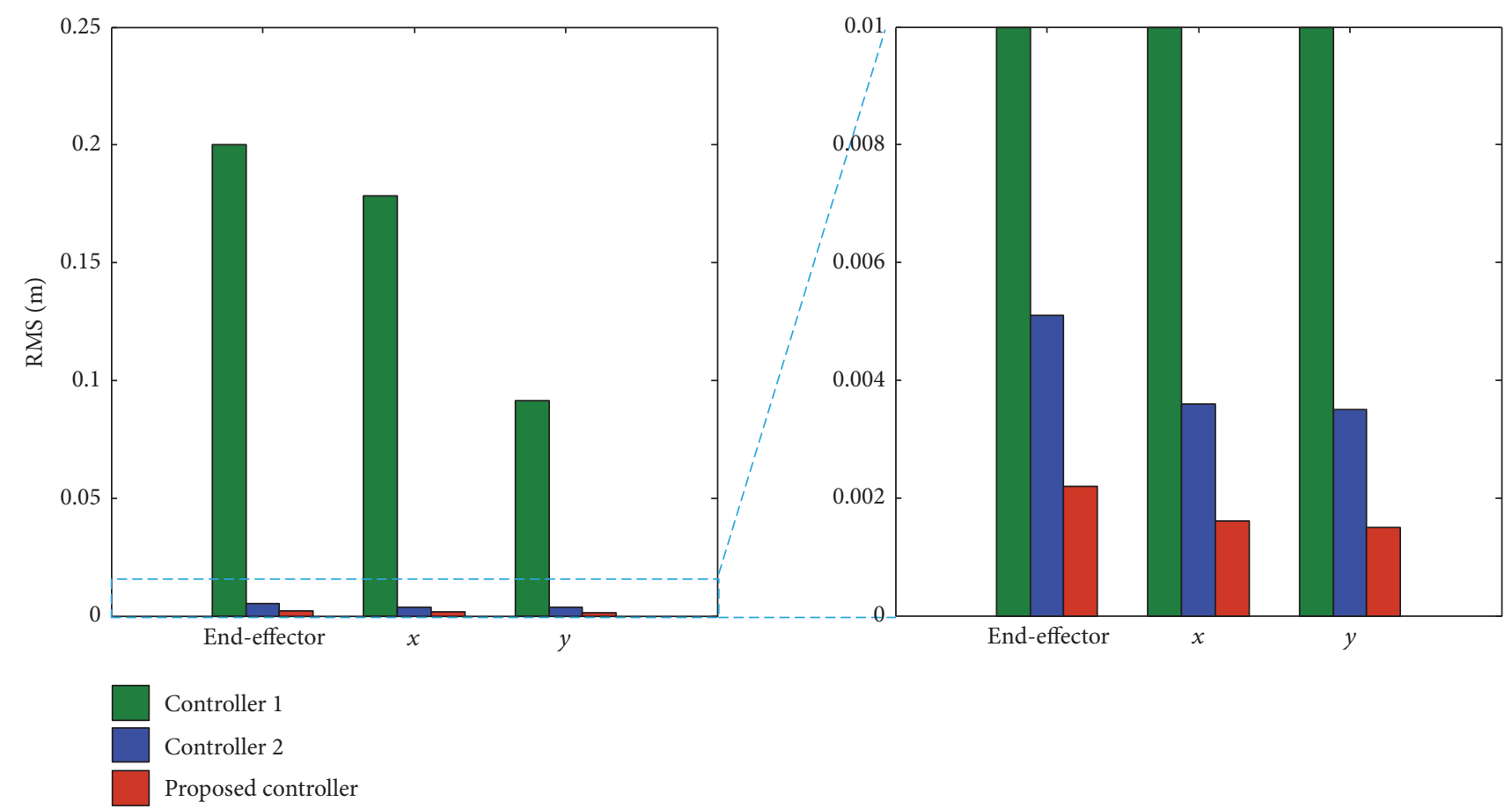

FIGURE 16: RMS of tracking errors with dynamic uncertainties.

convergence rate among the three controllers. As shown in Figures 15 and 16, the MAX of proposed controller is 3.1\% and $60.5 \%$ of those from other two controllers during the tracking error of end-effector. The RMS of proposed controller is $1.1 \%$ and $43.1 \%$ of those from other two controllers.
4.2.3. Case 3 with Measurement Noise. In practical applications, the measurement noise is unavoidable. Thus, to prove the practicality of the proposed controller, the measurement noise is introduced into the position measurement of robotic manipulator based on the above simulation. A band-limited 


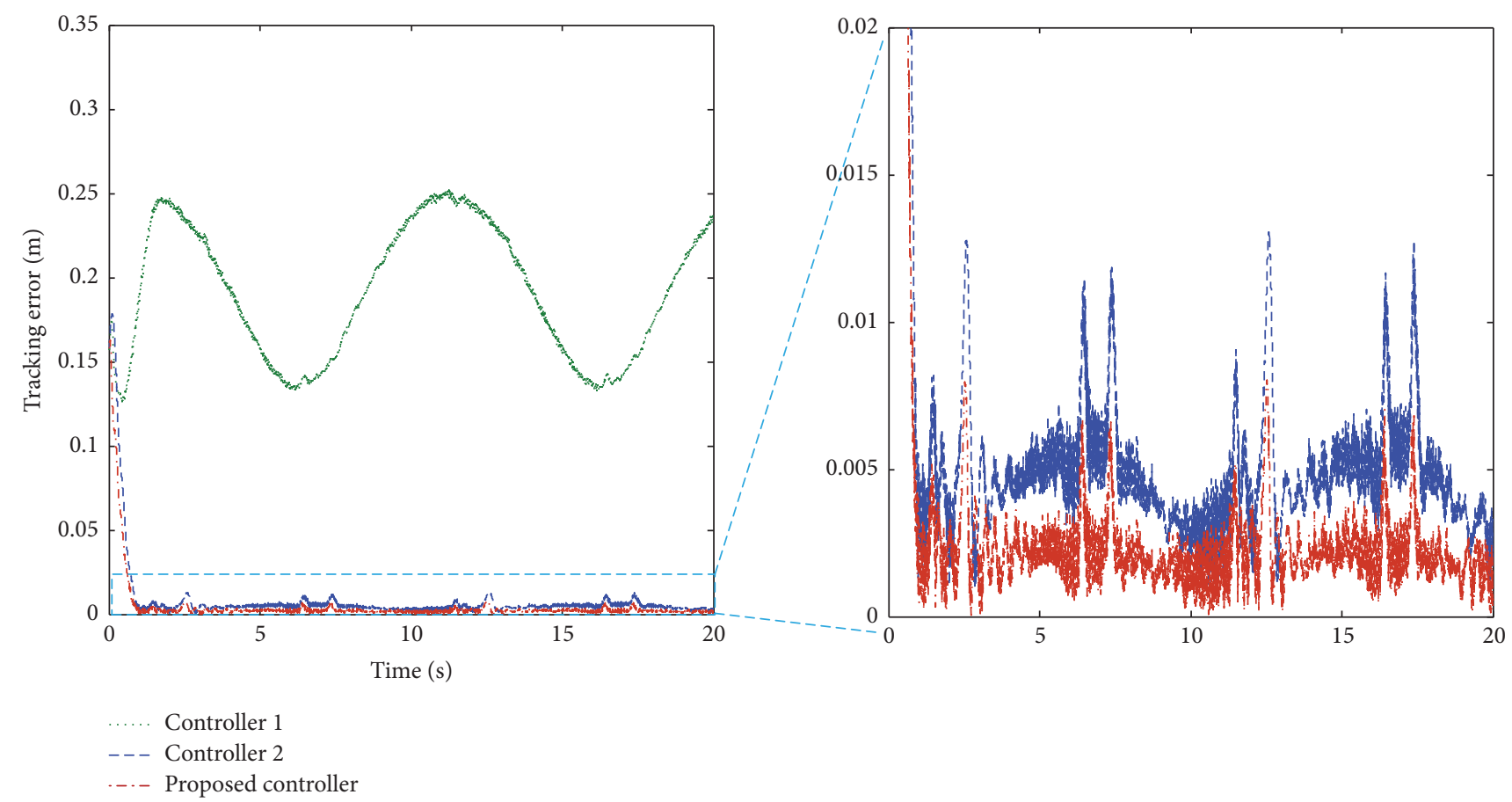

FIGURE 17: Tracking error of manipulator end-effector with measurement noise.

white noise module is used as the noise effect. The noise power is set as $4 \times 10^{-8}$, and other parameters remain at default values. After being filtered by the discrete filter module, the simulation results are shown in Figures 17-20.

As shown in Figures 17, 18, and 20, controller 2 and the proposed controller can ensure small tracking error under measurement noise. Controller 1 has a relative large tracking error due to the estimation of $l_{1}$ and $l_{2}$. The control input of the joint becomes noisy due to measurement noise. From the tracking result, the proposed controller still has the best control performance among the controllers. Moreover, it can be observed that the MAX and RMX of tracking errors of the proposed controller are smallest.

In summary, from those simulation results, it can be concluded that the proposed control ensures faster convergence rate and higher tracking precision under external kinematic and dynamic uncertainties. Furthermore, due to the application of TDE, the proposed controller is easy to implement and it does not require the dynamical model of robot system.

It is estimated that the good performance results from the controller structure closely. First, the controller considers the external kinematic uncertainties during the design procedure. Thus, the controller is not sensitive to the varying of kinematic uncertainties. The effectiveness of this consideration is showed by the comparison between controller 1 and the proposed controller. Second, TDE cancels most of the kinematic and dynamic uncertainties by the scheme $\boldsymbol{\tau}_{t-L}-\overline{\mathbf{M}} \ddot{\boldsymbol{\theta}}_{t-L}$. No prior knowledge of the robot dynamic and offline identification is required with TDE, which ensures the controller to be simple and easy to implement. Third, the high tracking precision is benefited from the NTSM and adaptive fuzzy logic scheme. NTSM is selected as the desired error dynamics, which improves the tracking precision and convergence rate. The implanting of fuzzy logic control scheme can eliminate the chattering caused by NTSM. Some peaks can be observed in the tracking error, which is caused by the by Coulomb friction when the sign of velocity changes. This scheme can compensate the TDE error effectively. Furthermore, it can also compensate the external kinematic and dynamic uncertainties. The advantage of using this scheme is verified by the comparison between controller 2 and the proposed controller.

It should be pointed out that simplicity is the critical virtue of the TDE based controllers. The combination of adaptive fuzzy logic scheme may introduce more parameters. The consideration about this problem in our controller design is discussed as follows. In the design process, adaptive scheme is implemented in the controller, which has the following advantages. First, the parameters of the factors in fuzzy mechanism and the compensator output value are adapted online. Second, the rule number in the FLC is reduced using the adaptive fuzzy nonsingular terminal sliding mode. Third, kinematic parameters of the system are not required exactly. Thus, with the above advantages, only one more parameter is introduced compared with the number of parameters in TDE based NTSM. The parameter tuning step is similar to TDE based NTSM. First, the parameters in terminal sliding mode should be selected. Then, the parameter $\overline{\mathbf{M}}$ can be tuned from small positive value to obtain a satisfactory control performance. After that, one can tune the three parameters in adaptive scheme to achieve greater performance. Since the tuning process of the controller is by trial and error method, further research will focus on the automatic tuning of controller parameters. 

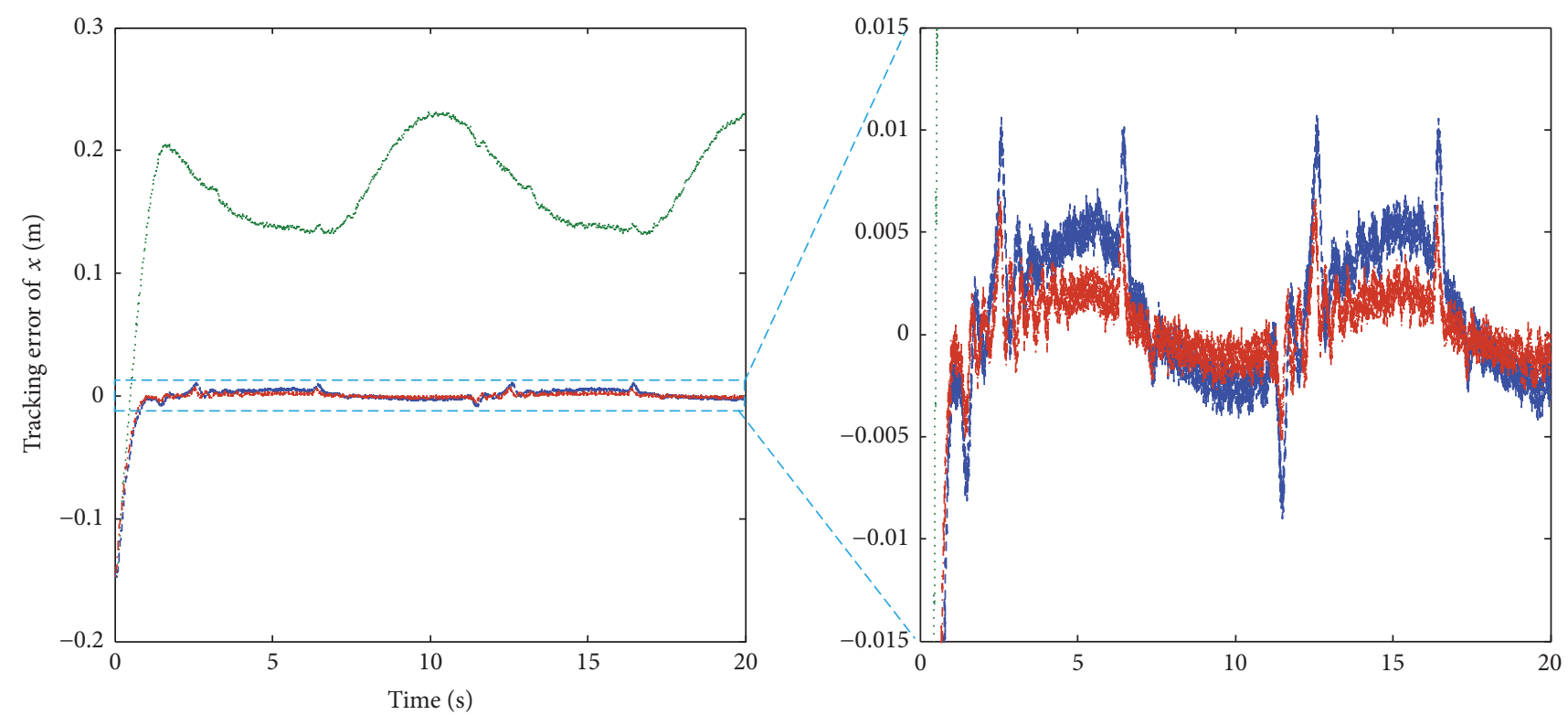

…. Controller 1

-- - Controller 2

... Proposed controller

(a)



(b)

FIGURE 18: Tracking error of $x$ and $y$ directions with measurement noise.

\section{Conclusions}

In this paper, a new control method is proposed for the trajectory tracking of robot manipulators in the task space under uncertain kinematics and dynamics. The controller is designed based on TDE. Kinematic parameters and dynamic models or numerous parameters of the robot manipulator systems are not exactly required in this controller. TDE scheme is used to cancel most of the uncertainties. The NTSM scheme endows desired error dynamics to provide finite time convergence. Adaptive fuzzy logic scheme is used to compensate the TDE error and eliminate the 


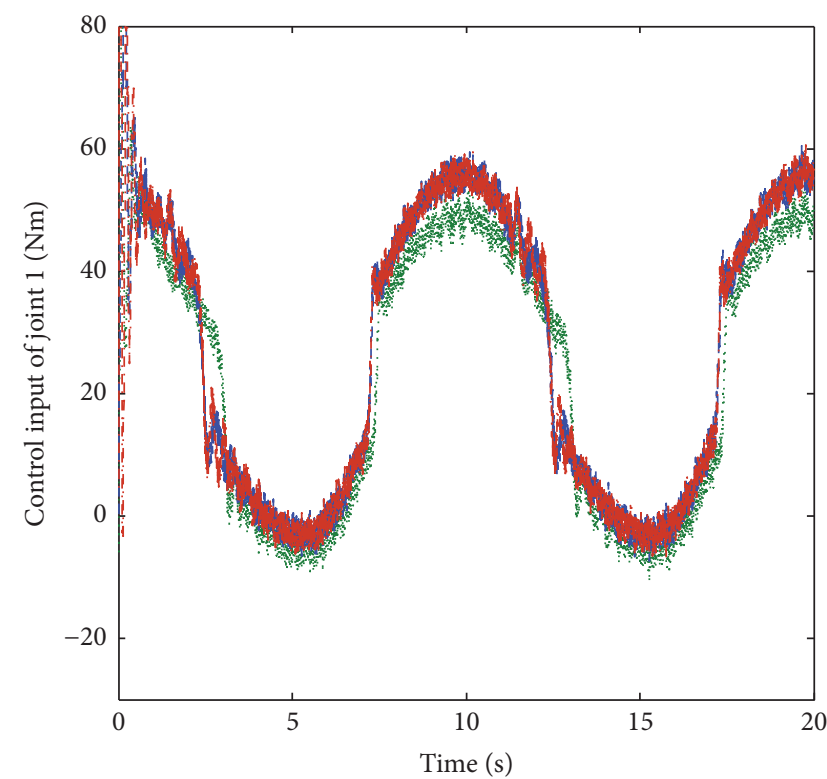

.... Controller 1

- - Controller 2

...- Proposed controller

(a)

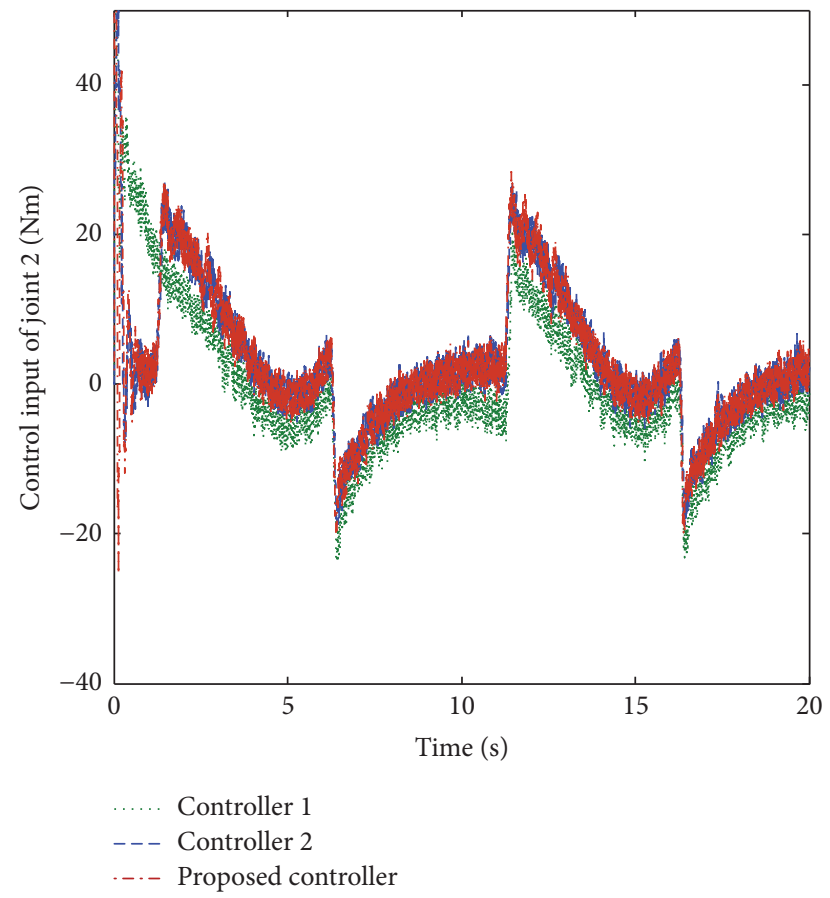

(b)

FIGURE 19: Control input of joint 1 and joint 2 with measurement noise.

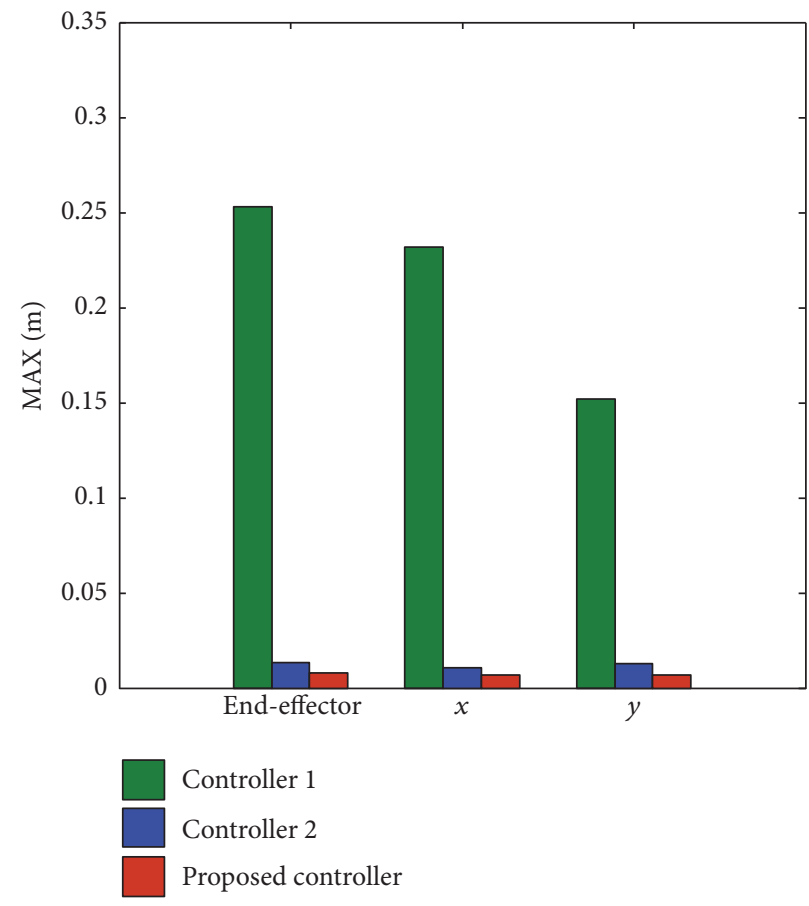

(a)

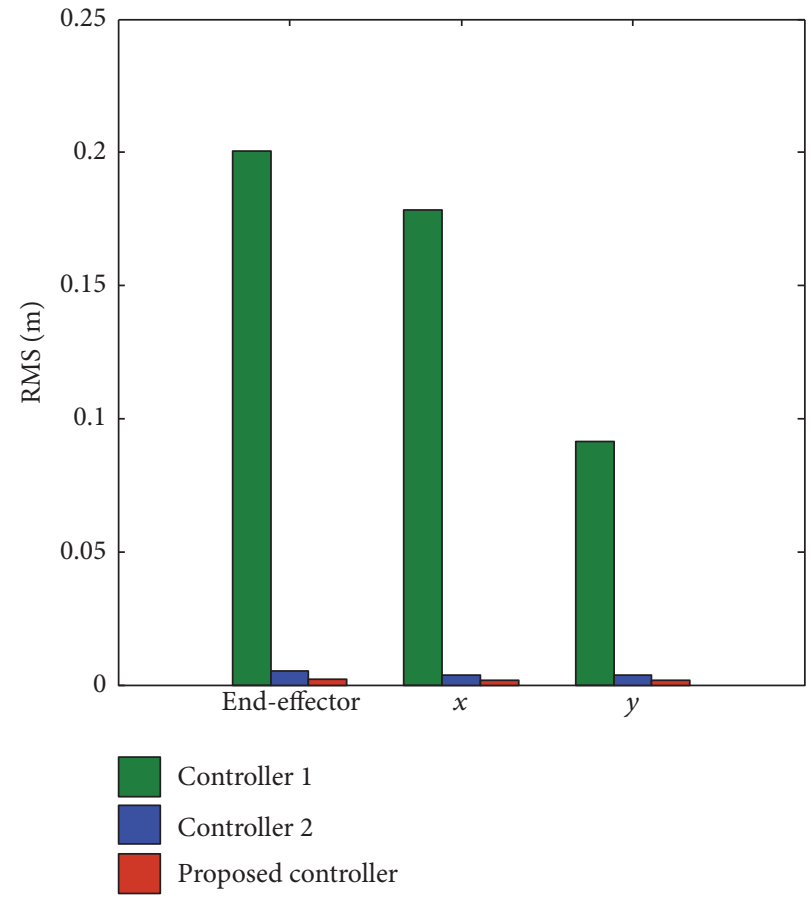

(b)

FIgURE 20: MAX and RMS of tracking errors with measurement noise.

chattering, which reduces the tracking error. The performance of proposed controller is verified through simulation on a 2-DOF robot manipulator. Three cases simulations are designed to track the desired trajectory in the task space using the controllers. The comparison results show that the proposed controller provides faster convergence rate and higher tracking precision than TDE based NTSM and improved TDE based NTSM controller. Furthermore, 
the controller is simple structure and easy to implement in practical applications. The automatic tuning of controller parameters and experiments verification of the proposed controller will be studied in the further research.

\section{Conflicts of Interest}

The authors declare that there are no conflicts of interest regarding the publication of this paper.

\section{Acknowledgments}

This project was funded by National Natural Science Foundation of China (51575320).

\section{References}

[1] W. He, A. O. David, Z. Yin, and C. Sun, "Neural network control of a robotic manipulator with input deadzone and output constraint," IEEE Transactions on Systems, Man, and Cybernetics: Systems, 2015.

[2] R. Sharma, K. P. S. Rana, and V. Kumar, "Performance analysis of fractional order fuzzy PID controllers applied to a robotic manipulator," Expert Systems with Applications, vol. 41, no. 9, pp. 4274-4289, 2014.

[3] J. Wilson, M. Charest, and R. Dubay, "Non-linear model predictive control schemes with application on a 2 link vertical robot manipulator," Robotics and Computer-Integrated Manufacturing, vol. 41, pp. 23-30, 2016.

[4] L. M. Capisani and A. Ferrara, "Trajectory planning and second-order sliding mode motion/interaction control for robot manipulators in unknown environments," IEEE Transactions on Industrial Electronics, vol. 59, no. 8, pp. 3189-3198, 2012.

[5] W. Lv, L. Tao, and Z. Ji, "Sliding mode control of cable-driven redundancy parallel robot with 6 DOF based on cable-length sensor feedback," Mathematical Problems in Engineering, Art. ID 1928673, 21 pages, 2017.

[6] H. Liu and T. Zhang, "Fuzzy sliding mode control of robotic manipulators with kinematic and dynamic uncertainties," Journal of Dynamic Systems, Measurement, and Control, vol. 134, no. 6, Article ID 061007, 2012.

[7] B. Xiao, S. Yin, and O. Kaynak, "Tracking Control of Robotic Manipulators with Uncertain Kinematics and Dynamics," IEEE Transactions on Industrial Electronics, vol. 63, no. 10, pp. 64396449, 2016.

[8] Q. Zhou, H. Li, and P. Shi, "Decentralized adaptive fuzzy tracking control for robot finger dynamics," IEEE Transactions on Fuzzy Systems, vol. 23, no. 3, pp. 501-510, 2015.

[9] S. I. Han and J. M. Lee, "Fuzzy echo state neural networks and funnel dynamic surface control for prescribed performance of a nonlinear dynamic system," IEEE Transactions on Industrial Electronics, vol. 61, no. 2, pp. 1099-1112, 2014.

[10] C. R. Kumar, K. R. Sudha, and D. V. Pushpalatha, "Design of Prisoner's dilemma based fuzzy logic computed torque controller with Lyapunov synthesis linguistic model for PUMA560 robot manipulator," Journal of Intelligent \& Fuzzy Systems: Applications in Engineering and Technology, vol. 31, no. 1, pp. 345-355, 2016.
[11] Y. Wang, L. Gu, Y. Xu, and X. Cao, "Practical tracking control of robot manipulators with continuous fractional-order nonsingular terminal sliding mode," IEEE Transactions on Industrial Electronics, vol. 63, no. 10, pp. 6194-6204, 2016.

[12] Y. Wang, L. Gu, B. Chen, and H. Wu, "A new discrete time delay control of hydraulic manipulators," Proceedings of the Institution of Mechanical Engineers, Part I: Journal of Systems and Control Engineering, vol. 231, no. 3, pp. 168-177, 2017.

[13] S. Roy, I. N. Kar, J. Lee, and M. Jin, "Adaptive-Robust TimeDelay Control for a Class of Uncertain Euler-Lagrange Systems," IEEE Transactions on Industrial Electronics, vol. 64, no. 9, pp. 7109-7119, 2017.

[14] S.-J. Cho, M. Jin, T.-Y. Kuc, and J. S. Lee, "Control and synchronization of chaos systems using time-delay estimation and supervising switching control," Nonlinear Dynamics, vol. 75, no. 3, pp. 549-560, 2014.

[15] J. Lee, C. Yoo, Y.-S. Park et al., "An experimental study on time delay control of actuation system of tilt rotor unmanned aerial vehicle," Mechatronics, vol. 22, no. 2, pp. 184-194, 2012.

[16] J. Lee, M. Jin, and K. K. Ahn, "Precise tracking control of shape memory alloy actuator systems using hyperbolic tangential sliding mode control with time delay estimation," Mechatronics, vol. 23, no. 3, pp. 310-317, 2013.

[17] J. Lee, P. H. Chang, and M. Jin, "Adaptive integral sliding mode control with time-delay estimation for robot manipulators," IEEE Transactions on Industrial Electronics, vol. 64, no. 8, pp. 6796-6804, 2017.

[18] J. Kim, P. Chang, and M. Jin, "Fuzzy PID controller design using time-delay estimation," Transactions of the Institute of Measurement and Control, vol. 39, no. 9, pp. 1329-1338, 2016.

[19] S.-U. Lee and P. H. Chang, "Control of a heavy-duty robotic excavator using time delay control with integral sliding surface," Control Engineering Practice, vol. 10, no. 7, pp. 697-711, 2002.

[20] H. J. Bae, M. Jin, J. Suh, J. Y. Lee, P. H. Chang, and D. S. Ahn, "Control of robot manipulators using time-delay estimation and fuzzy logic systems," Journal of Electrical Engineering Technology, vol. 12, no. 3, pp. 1271-1279, 2017.

[21] M. Jin, J. Lee, and N. G. Tsagarakis, "Model-free robust adaptive control of humanoid robots with flexible joints," IEEE Transactions on Industrial Electronics, vol. 64, no. 2, pp. 17061715, 2017.

[22] S. Cho, J. S. Lee, J. Kim, T. Kuc, P. Chang, and M. Jin, "Adaptive time-delay control with a supervising switching technique for robot manipulators," Transactions of the Institute of Measurement and Control, vol. 39, no. 9, pp. 1374-1382, 2016.

[23] M. Jin, Y. Jin, P. H. Chang, and C. Choi, "High-accuracy tracking control of robot manipulators using time delay estimation and terminal sliding mode," International Journal of Advanced Robotic Systems, vol. 8, no. 4, pp. 65-78, 2011.

[24] M. Jin, J. Lee, P. H. Chang, and C. Choi, "Practical nonsingular terminal sliding-mode control of robot manipulators for highaccuracy tracking control," IEEE Transactions on Industrial Electronics, vol. 56, no. 9, pp. 3593-3601, 2009.

[25] M. Jin, S. H. Kang, and P. H. Chang, "Robust compliant motion control of robot with nonlinear friction using timedelay estimation," IEEE Transactions on Industrial Electronics, vol. 55, no. 1, pp. 258-269, 2008.

[26] G. R. Cho, P. H. Chang, S. H. Park, and M. Jin, "Robust tracking under nonlinear friction using time-delay control with internal model," IEEE Transactions on Control Systems Technology, vol. 17, no. 6, pp. 1406-1414, 2009. 
[27] D. K. Han and P.-H. Chang, "Robust tracking of robot manipulator with nonlinear friction using time delay control with gradient estimator," Journal of Mechanical Science and Technology, vol. 24, no. 8, pp. 1743-1752, 2010.

[28] D. Oetomo and M. H. Ang Jr., "Singularity robust algorithm in serial manipulators," Robotics and Computer-Integrated Manufacturing, vol. 25, no. 1, pp. 122-134, 2009.

[29] Y. Guo and P.-Y. Woo, "An adaptive fuzzy sliding mode controller for robotic manipulators," IEEE Transactions on Systems, Man and Cybernetics, Part A: Systems and Humans, vol. 33, no. 2, pp. 149-159, 2003.

[30] M. R. Soltanpour and M. M. Fateh, "Sliding mode robust control of robot manipulator in the task space by support of Feedback Linearization and Back Stepping Control," World Applied Sciences Journal, vol. 6, no. 1, pp. 70-76, 2009. 


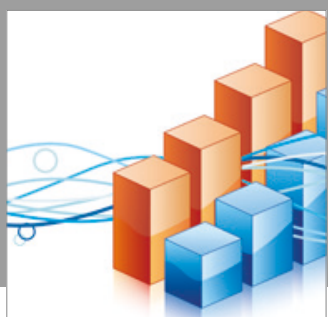

Advances in

Operations Research

vatersals

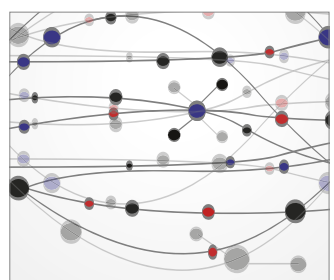

\section{The Scientific} World Journal
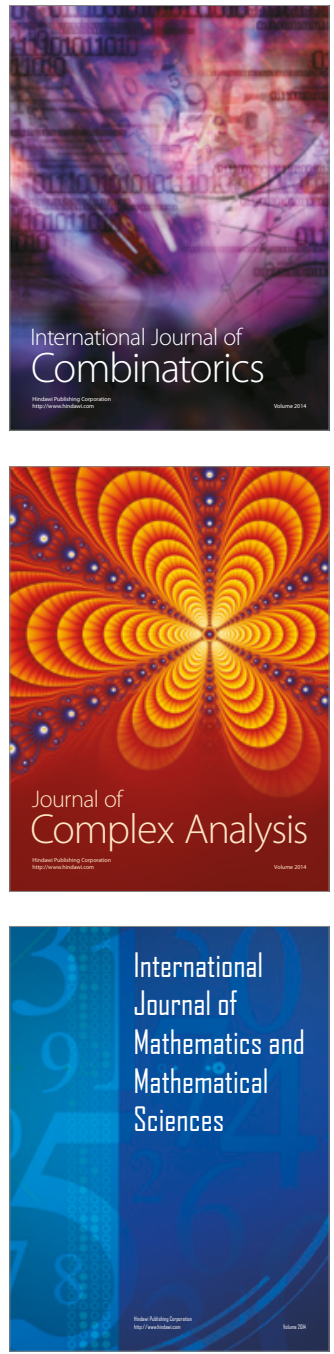
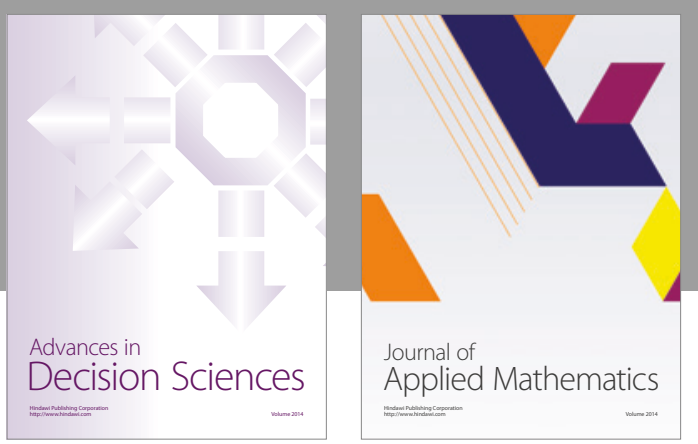

Algebra

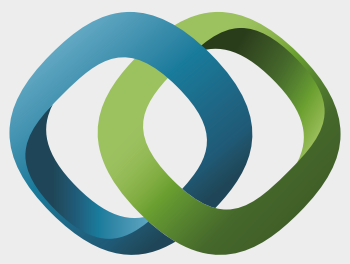

\section{Hindawi}

Submit your manuscripts at

https://www.hindawi.com
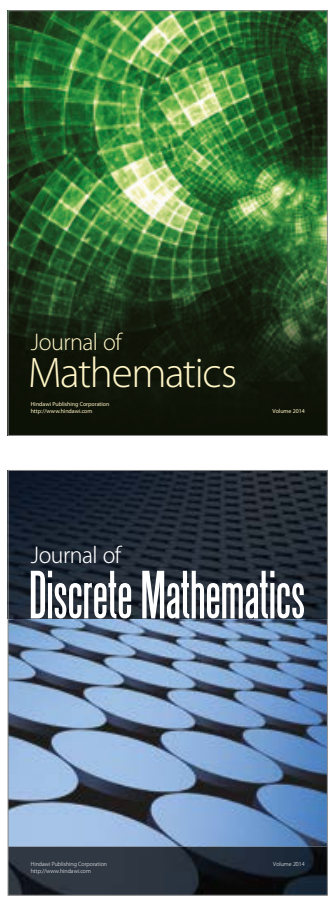

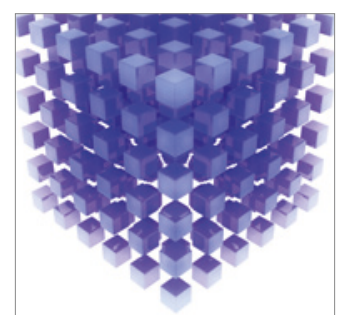

Mathematical Problems in Engineering
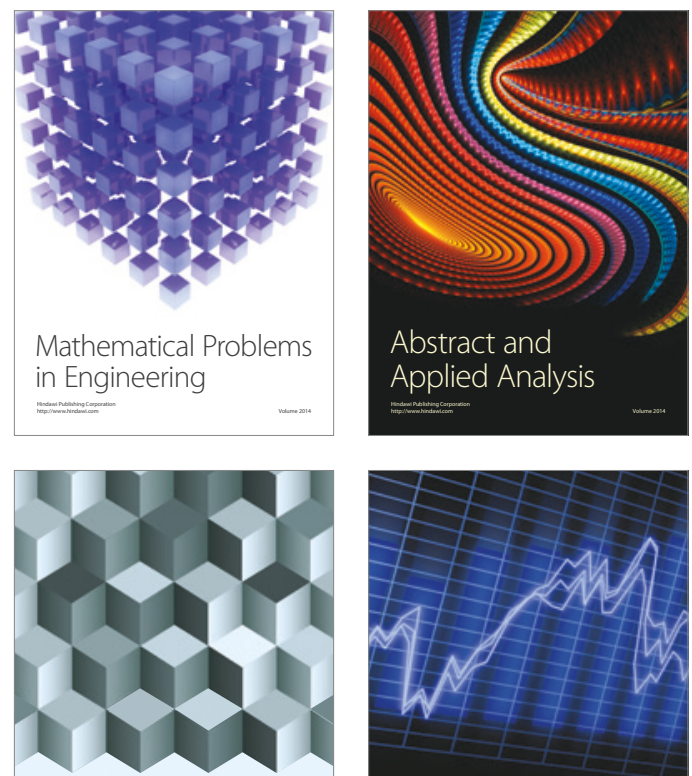

Journal of

Function Spaces

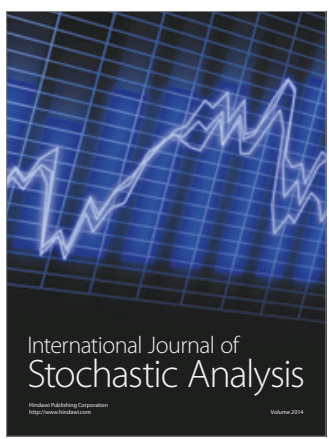

Probability and Statistics
